\title{
Asymptotic Stability of Combination of Contact Discontinuity with Rarefaction Waves for the One-Dimensional Viscous Micropolar Fluid Model
}

\author{
Lishuang Peng \\ College of Science, University of Shanghai for Science and Technology, Shanghai, China \\ Email: penglishuang1@163.com
}

How to cite this paper: Peng, L.S. (2019) Asymptotic Stability of Combination of Contact Discontinuity with Rarefaction Waves for the One-Dimensional Viscous Micropolar Fluid Model. Journal of Applied Mathematics and Physics, 7, 2089-2111. https://doi.org/10.4236/jamp.2019.79143

Received: August 23, 2019

Accepted: September 21, 2019

Published: September 24, 2019

Copyright $\odot 2019$ by author(s) and Scientific Research Publishing Inc. This work is licensed under the Creative Commons Attribution International License (CC BY 4.0).

http://creativecommons.org/licenses/by/4.0/

\begin{abstract}
In this paper, we consider with the large time behavior of solutions of the Cauchy problem to the one-dimensional compressible micropolar fluid model, where the far field states are prescribed. When the corresponding Riemann problem for the Euler system admits the solution consisting of contact discontinuity and rarefaction waves, it is shown that the combination wave corresponding to the contact discontinuity, with rarefaction waves is asymptotically stable provided that the strength of the combination wave and the initial perturbation are suitably small. This result is proved by using elementary $L^{2}$-energy methods.
\end{abstract}

\section{Keywords}

Contact Discontinuity, Rarefaction Waves, Viscous Micropolar Fluid Model, Asymptotic Stability, Energy Estimates

\section{Introduction}

In this paper, we consider the one-dimensional viscous micropolar fluid model in Lagrangian coordinates:

$$
\left\{\begin{array}{l}
v_{t}-u_{x}=0, \\
u_{t}+p_{x}=\mu\left(\frac{u_{x}}{v}\right)_{x}, \\
\left(e+\frac{u^{2}}{2}\right)_{t}+(p u)_{x}=\kappa\left(\frac{\theta_{x}}{v}\right)_{x}+\mu\left(\frac{u u_{x}}{v}\right)_{x}+\frac{\omega_{x}^{2}}{v}+v \omega^{2}, \\
\omega_{t}=A\left[\left(\frac{\omega_{x}}{v}\right)_{x}-v \omega\right] .
\end{array}\right.
$$


Here $x \in \mathbb{R}$ is the Lagrangian space variable, $t \in \mathbb{R}^{+}$the time variable and the primary dependent variable are the specific volume $v=v(t, x)>0$, the velocity $u=u(t, x)$, the absolute temperature $\theta=\theta(t, x)>0$ and the microrotation velocity $\omega=\omega(t, x)$. The positive constants $\mu, \kappa$ and $A$ denote the viscosity coefficient, heat conduction coefficient and microviscosity coefficient, respectively. The pressure $p$ and the internal energy $e$ are given by the state equations

$$
p(v, \theta)=\frac{R \theta}{v}=B v^{-\gamma} \exp \left(\frac{\gamma-1}{R} s\right), \quad e=\frac{R}{\gamma-1} \theta,
$$

where $\gamma>1$ is the adiabatic exponent, $s$ is the entropy of fluid, $R$ and $B$ are the positive constants. We impose the following initial and far field conditions:

$$
\left\{\begin{array}{l}
(v, u, \theta, \omega)(x, 0)=\left(v_{0}, u_{0}, \theta_{0}, \omega_{0}\right)(x) \quad x \in \mathbb{R}, \\
(v, u, \theta, \omega)( \pm \infty, 0)=\left(v_{ \pm}, u_{ \pm}, \theta_{ \pm}, \omega_{ \pm}\right)(x) \quad t>0 .
\end{array}\right.
$$

where $v_{ \pm}>0, u_{ \pm}, \theta_{ \pm}>0, \omega_{ \pm}$are given constants, and we assume $\inf _{\mathbb{R}} v_{0}>0$, $\inf _{\mathbb{R}} \theta_{0}>0,\left(v_{0}, u_{0}, \theta_{0}, \omega_{0}\right)( \pm \infty)=\left(v_{ \pm}, u_{ \pm}, \theta_{ \pm}, \omega_{ \pm}\right)$as compatibility conditions.

It is known that the large-time behavior of solutions of the Cauchy problem (1) and (2) is closely related to the Riemann problem of the compressible Euler system:

$$
\left\{\begin{array}{l}
v_{t}-u_{x}=0, \\
u_{t}+p(v, \theta)_{x}=0, \quad x \in \mathbb{R}, t>0 . \\
\left(e+\frac{u^{2}}{2}\right)_{t}+(p u)_{x}=0,
\end{array}\right.
$$

with the Riemann initial data

$$
(v, u, \theta)(0, x)= \begin{cases}\left(v_{-}, u_{-}, \theta_{-}\right), & x<0, \\ \left(v_{+}, u_{+}, \theta_{+}\right), & x>0 .\end{cases}
$$

It is well-known that the above system has three eigenvalues:

$$
\lambda_{1}(v, \theta)=-\sqrt{\frac{\gamma p}{v}}, \quad \lambda_{2}=0, \quad \lambda_{3}(v, \theta)=\sqrt{\frac{\gamma p}{v}},
$$

which implies that the first and third characteristic fields are genuinely nonlinear and the second field is linearly degenerate. Then it is known that the basic Riemann solutions of the problem (3)-(4) are dilation invariant solutions: shock waves, rarefaction waves, contact discontinuities, and the linear combinations of these basic waves. In particular, the contact discontinuity solution of the Riemann problems (3) and (4) takes the form [1]:

$$
\left(v^{c d}, u^{c d}, \theta^{c d}\right)(t, x)= \begin{cases}\left(v_{-}, u_{-}, \theta_{-}\right), & x<0, t>0, \\ \left(v_{+}, u_{+}, \theta_{+}\right), & x>0, t>0,\end{cases}
$$

provided that

$$
u_{-}=u_{+}, \quad p_{-} \triangleq \frac{R \theta_{-}}{v_{-}}=\frac{R \theta_{+}}{v_{+}} \triangleq p_{+} .
$$

The viscous contact wave $\left(V^{c}, U^{c}, \Theta^{c}, W^{c}\right)(t, x)$ with $W^{c}(t, x)=0$ corres- 
ponding to the contact discontinuity $\left(v^{c d}, u^{c d}, \theta^{c d}, \omega^{c d}\right)(t, x)$ with $\left(v^{c d}, u^{c d}, \theta^{c d}\right)$ defined in (5) and $\omega^{c d}(t, x)=0$ for the compressible micropolar fluid model (1) becomes smooth and behaviors as a diffusion waves due to the effect of heat conductivity. As in [2], we can define the viscous contact wave $\left(V^{c}, U^{c}, \Theta^{c}\right)(t, x)$ as follows.

Since the pressure of the profile $\left(V^{c}, U^{c}, \Theta^{c}\right)(t, x)$ is expected to be almost constant, we set

$$
P^{c} \equiv \frac{R \Theta^{c}}{V^{c}} \approx p_{+}=p_{-} .
$$

Then the leading part of the energy Equation $(1)_{3}$ is

$$
\frac{R}{\gamma-1} \Theta_{t}^{c}+p_{+} U_{x}^{c}=\kappa\left(\frac{\Theta_{x}^{c}}{V^{c}}\right)_{x} .
$$

Using the Equation (7), $V_{t}^{c}=U_{x}^{c}$ and (8), we get a nonlinear diffusion equation

$$
\Theta_{t}^{c}=a\left(\frac{\Theta_{x}^{c}}{\Theta^{c}}\right)_{x}, \quad \Theta^{c}( \pm \infty, t)=\theta_{ \pm}, \quad a=\frac{\kappa p_{+}(\gamma-1)}{\gamma R^{2}}>0,
$$

which has a unique self-similar solution $\Theta^{c}(t, x)=\Theta^{c}(\xi), \xi=\frac{x}{\sqrt{1+t}}$ because of [3] [4]. Furthermore, $\Theta(\xi)$ is a monotone function, increasing if $\theta_{+}>\theta_{-}$ and decreasing if $\theta_{+}<\theta_{-}$. On the other hand, there exists some positive constant $\tilde{\delta}$, such that for $\delta=\left|\theta_{+}-\theta_{-}\right| \leq \tilde{\delta}, \Theta^{c}$ satisfies

$$
(1+t)^{\frac{k}{2}}\left|\partial_{x}^{k} \Theta^{c}\right|+\left|\Theta^{c}-\theta_{ \pm}\right| \leq c_{1} \delta \mathrm{e}^{-\frac{c_{0} x^{2}}{1+t}}, k \geq 1, \text { as }|x| \rightarrow \infty,
$$

where $c_{0}$ and $c_{1}$ are two positive constants depending only on $\theta_{-}$and $\tilde{\delta}$. Once $\Theta^{c}(t, x)$ is determined, the viscous contact wave profile $\left(V^{c}, U^{c}, \Theta^{c}\right)(t, x)$ is defined as follows:

$$
V^{c}=\frac{R \Theta^{c}}{p_{+}}, U^{c}=u_{-}+\frac{\kappa(\gamma-1)}{\gamma R} \frac{\Theta_{x}^{c}}{\Theta^{c}}, \Theta^{c}=\Theta^{c}(t, x) .
$$

It is straightforward to check that $\left(V^{c}, U^{c}, \Theta^{c}\right)$ satisfies

$$
\left\|V^{c}-v^{c d}, U^{c}-u^{c d}, \Theta^{c}-\theta^{c d}\right\|_{L^{p}}=O\left(\kappa^{1 /(2 p)}\right)(1+t)^{1 /(2 p)}, p \geq 1,
$$

which means the nonlinear diffusion wave $\left(V^{c}, U^{c}, \Theta^{c}\right)(x, t)$ approximates the contact discontinuity $\left(v^{c d}, u^{c d}, \theta^{c d}\right)(x, t)$ to the Euler system (3) in $L^{p}$ norm, $p \geq 1$ on any finite time interval as the heat conductivity coefficient $\kappa$ tends to zero. And it is easy to check that the viscous contact wave $\left(V^{c}, U^{c}, \Theta^{c}\right)$ satisfies the system

$$
\left\{\begin{array}{l}
V_{t}^{c}-U_{x}^{c}=0, \\
U_{t}^{c}+\left(\frac{R \Theta^{c}}{V^{c}}\right)_{x}=\mu\left(\frac{U_{x}^{c}}{V^{c}}\right)_{x}+R_{1}, \\
\left(e\left(V^{c}, \Theta^{c}\right)+\frac{\left(U^{c}\right)^{2}}{2}\right)_{t}+\left(p\left(V^{c}, \Theta^{c}\right) U^{c}\right)_{x}=\left(\kappa \frac{\Theta_{x}^{c}}{V^{c}}+\mu \frac{U^{c} U_{x}^{c}}{V^{c}}\right)_{x}+R_{2},
\end{array}\right.
$$


where

$$
\begin{aligned}
R_{1} & =U_{t}^{c}-\mu\left(\frac{U_{x}^{c}}{V^{c}}\right)_{x}=\frac{\kappa(\gamma-1)}{\gamma R}\left(\left(\ln \Theta^{c}\right)_{x t}-\mu\left(\frac{p_{+}}{R \Theta^{c}}\left(\ln \Theta^{c}\right)_{x x}\right)_{x}\right) \\
& =O(\delta)(1+t)^{-\frac{3}{2}} \mathrm{e}^{-\frac{c_{0} x^{2}}{1+t}} \text { as }|x| \rightarrow \infty,
\end{aligned}
$$

and

$$
\begin{aligned}
R_{2} & =U^{c} U_{t}^{c}-\mu\left(\frac{U^{c} U_{x}^{c}}{V^{c}}\right)_{x} \\
& =\left(\frac{\kappa(\gamma-1)}{\gamma R}\right)^{2}\left(\left(\ln \Theta^{c}\right)_{x}\left(\ln \Theta^{c}\right)_{x t}-\mu\left(\frac{p_{+}}{R \Theta^{c}}\left(\ln \Theta^{c}\right)_{x}\left(\ln \Theta^{c}\right)_{x x}\right)_{x}\right) \\
& =O(\delta)(1+t)^{-2} \mathrm{e}^{-\frac{c_{0} x^{2}}{1+t}} \text { as }|x| \rightarrow \infty .
\end{aligned}
$$

We are now in a position to state our main results. Let

$$
(\phi, \psi, \zeta, \omega)(x, t)=\left(v-V^{c}, u-U^{c}, \theta-\Theta^{c}, \omega\right)(t, x) .
$$

For interval $I \subset[0, \infty)$, we define a function space

$$
X(I) \subset Y(I) \triangleq C\left(I ; H^{1}(\mathbb{R})\right)
$$

as

$$
X(I)=\left\{(\phi, \psi, \zeta, \omega) \in Y(I) \mid \phi_{x} \in L^{2}\left(I ; L^{2}(\mathbb{R})\right),\left(\psi_{x}, \zeta_{x}, \omega_{x}\right) \in L^{2}\left(I ; H^{1}(\mathbb{R})\right)\right\} .
$$

We can get main result from [5] and it is stated as the following theorem.

Theorem 1.1. For any given $\left(v_{-}, u_{-}, \theta_{-}\right)$, suppose that $\left(v_{+}, u_{+}, \theta_{+}\right)$satisfies (6), Let $\left(V^{c}, U^{c}, \Theta^{c}\right)(t, x)$ be the viscous contact wave defined in (11) with strength $\delta=\left|\theta_{+}-\theta_{-}\right| \leq \tilde{\delta}$. Then there exist positive constants $\delta_{0}$ and $\varepsilon_{0}$, such that if $\delta<\delta_{0}$ and the initial data $\left(v_{0}, u_{0}, \theta_{0}, \omega_{0}\right)$ satisfies

$$
\left\|\left(v_{0}(\cdot)-V^{c}(0, \cdot), u_{0}(\cdot)-U^{c}(0, \cdot), \theta_{0}(\cdot)-\Theta^{c}(0, \cdot), \omega_{0}(\cdot)\right)\right\|_{H^{1}(\mathbb{R})} \leq \varepsilon_{0},
$$

then the Cauchy problem (1)-(2) admits a unique global solution $(v, u, \theta, \omega)(t, x)$ satisfying $\left(v-V^{c}, u-U^{c}, \theta-\Theta^{c}, \omega\right)(t, x) \in X([0, \infty))$ and

$$
\limsup _{t \in \infty}\left\{\left\{\left(v-V^{c}, u-U^{c}, \theta-\Theta^{c}, \omega\right)(t, x) \mid\right\}=0 .\right.
$$

When the relation (6) fails, the basic theory of hyperbolic systems of conservation laws (for example, see [6]) implies that for any given constant state $\left(v_{-}, u_{-}, \theta_{-}\right)$with $v_{-}>0, \theta_{-}>0$ and $u_{-} \in \mathbb{R}$, there exists a suitable neighborhood $\Omega\left(v_{-}, u_{-}, \theta_{-}\right)$of $\left(v_{-}, u_{-}, \theta_{-}\right)$such that for any $\left(v_{+}, u_{+}, \theta_{+}\right) \in \Omega\left(v_{-}, u_{-}, \theta_{-}\right)$, the Riemann problem of the Euler system (3)-(4) has a unique solution. In this paper, we only consider the stability of the superposition of the viscous contact wave and rarefaction waves. In this situation, we assume that

$$
\left(v_{+}, u_{+}, \theta_{+}\right) \in R_{1} C R_{3}\left(v_{-}, u_{-}, \theta_{-}\right) \subset \Omega\left(v_{-}, u_{-}, \theta_{-}\right),
$$

where 


$$
\begin{aligned}
& R_{1} C R_{3}\left(v_{-}, u_{-}, \theta_{-}\right) \triangleq\left\{(u, v, \theta) \in \Omega\left(v_{-}, u_{-}, \theta_{-}\right) \mid s \neq s_{-},\right. \\
& \left.u \geq u_{-}-\int_{v_{-}}^{\frac{\gamma-1}{R \gamma}\left(s_{-}-s\right)} v \lambda_{-}\left(\eta, s_{-}\right) \mathrm{d} \eta, u \geq u_{-}-\int_{\mathrm{e}^{\frac{\gamma-1}{R \lambda}\left(s-s_{-}\right)_{v_{-}}}}^{v} \lambda_{+}(\eta, s) \mathrm{d} \eta\right\}
\end{aligned}
$$

with

$$
s=\frac{R}{\gamma-1} \ln \frac{R \theta}{B}+R \ln v, \quad s_{ \pm}=\frac{R}{\gamma-1} \ln \frac{R \theta_{ \pm}}{B}+R \ln v_{ \pm},
$$

and

$$
\lambda_{ \pm}(v, s)= \pm \sqrt{B \gamma v^{-\gamma-1} \mathrm{e}^{(\gamma-1) \frac{s}{R}}} .
$$

It is well-known [6] that there exists some suitably small $\delta_{1}>0$ such that for

$$
\left(v_{+}, u_{+}, \theta_{+}\right) \in R_{1} C R_{3}\left(v_{-}, u_{-}, \theta_{-}\right), \quad\left|\theta_{-}-\theta_{+}\right| \leq \delta_{1},
$$

there exists a positive constant $C=C\left(\theta_{-}, \delta_{1}\right)$ and a unique pair of points $\left(v_{-}^{m}, u^{m}, \theta_{-}^{m}\right)$ and $\left(v_{+}^{m}, u^{m}, \theta_{+}^{m}\right)$ in $\Omega\left(v_{-}, u_{-}, \theta_{-}\right)$satisfying

$$
\frac{R \theta_{-}^{m}}{v_{-}^{m}}=\frac{R \theta_{+}^{m}}{v_{+}^{m}} \triangleq p^{m}
$$

and

$$
\left|v_{ \pm}^{m}-v_{ \pm}\right|+\left|u^{m}-u_{ \pm}\right|+\left|\theta_{ \pm}^{m}-\theta_{ \pm}\right| \leq C\left|\theta_{-}-\theta_{+}\right| .
$$

Moreover, the points $\left(v_{-}^{m}, u^{m}, \theta_{-}^{m}\right)$ and $\left(v_{+}^{m}, u^{m}, \theta_{+}^{m}\right)$ belong to the 1-rarefaction wave curve $R_{-}\left(v_{-}, u_{-}, \theta_{-}\right)$and the 3 -rarefaction wave curve $R_{+}\left(v_{+}, u_{+}, \theta_{+}\right)$, respectively, where

$$
R_{ \pm}\left(v_{ \pm}, u_{ \pm}, \theta_{ \pm}\right)=\left\{s=s_{ \pm}, u=u_{ \pm}-\int_{v_{ \pm}}^{v} \lambda_{ \pm}\left(\eta, s_{ \pm}\right) \mathrm{d} \eta, v>v_{ \pm}\right\} .
$$

The points $\left(v_{-}^{m}, u^{m}, \theta_{-}^{m}\right)$ and $\left(v_{+}^{m}, u^{m}, \theta_{+}^{m}\right)$ may coincide with $\left(v_{-}, u_{-}, \theta_{-}\right)$ and $\left(v_{+}, u_{+}, \theta_{+}\right)$, respectively. The 1-rarefaction wave $\left(v_{-}^{r}, u_{-}^{r}, \theta_{-}^{r}\right)\left(\frac{x}{t}\right)$ (respectively the 3-rarefaction wave $\left.\left(v_{+}^{r}, u_{+}^{r}, \theta_{+}^{r}\right)\left(\frac{x}{t}\right)\right)$ connecting $\left(v_{-}, u_{-}, \theta_{-}\right)$and $\left(v_{-}^{m}, u^{m}, \theta_{-}^{m}\right)$ (respectively $\left(v_{+}^{m}, u^{m}, \theta_{+}^{m}\right)$ and $\left.\left(v_{+}, u_{+}, \theta_{+}\right)\right)$is the weak solution of the Riemann problem of the Euler system (3) with the following initial Riemann data

$$
\left(v_{ \pm}^{r}, u_{ \pm}^{r}, \theta_{ \pm}^{r}\right)(0, x)= \begin{cases}\left(v_{ \pm}^{m}, u^{m}, \theta_{ \pm}^{r}\right), & \pm x<0, \\ \left(v_{ \pm}, u_{ \pm}, \theta_{ \pm}\right), & \pm x>0 .\end{cases}
$$

Since the rarefaction waves $\left(v_{ \pm}^{r}, u_{ \pm}^{r}, \theta_{ \pm}^{r}\right)$ are not smooth enough solutions, it is convenient to construct smooth approximate ones. Motivated by [7], the smooth solutions of Euler system (3), $\left(V_{ \pm}^{r}, U_{ \pm}^{r}, \Theta_{ \pm}^{r}\right)$, which approximate $\left(v_{ \pm}^{r}, u_{ \pm}^{r}, \theta_{ \pm}^{r}\right)$ are given by 


$$
\left\{\begin{array}{l}
\lambda_{ \pm}\left(V_{ \pm}^{r}(t, x), s_{ \pm}\right)=\hat{\omega}_{ \pm}(t, x), \\
U_{ \pm}^{r}=u_{ \pm}-\int_{v_{ \pm}}^{V_{ \pm}^{r}(t, x)} \lambda_{ \pm}\left(\eta, s_{ \pm}\right) \mathrm{d} \eta, \\
\Theta_{ \pm}^{r}=\theta_{ \pm}\left(v_{ \pm}\right)^{\gamma-1}\left(V_{ \pm}^{r}\right)^{1-\gamma},
\end{array}\right.
$$

where $\hat{\omega}_{-}(t, x)$ (respectively $\left.\hat{\omega}_{+}(t, x)\right)$ is the solution of the initial problem for the typical Burgers equation:

$$
\left\{\begin{array}{l}
\hat{\omega}_{t}+\hat{\omega} \hat{\omega}_{x}=0, \quad x \in \mathbb{R}, t>0, \\
\hat{\omega}(0, x)=\frac{\hat{\omega}_{r}+\hat{\omega}_{l}}{2}+\frac{\hat{\omega}_{r}-\hat{\omega}_{l}}{2} \tanh x,
\end{array}\right.
$$

with $\hat{\omega}_{l}=\lambda_{-}\left(v_{-}, s_{-}\right)$and $\hat{\omega}_{r}=\lambda_{-}\left(v_{-}^{m}, s_{-}\right)$(respectively $\hat{\omega}_{l}=\lambda_{+}\left(v_{+}^{m}, s_{+}\right)$and $\left.\hat{\omega}_{r}=\lambda_{+}\left(v_{+}, s_{+}\right)\right)$.

Let $\left(V^{c}, U^{c}, \Theta^{c}\right)(t, x)$ be the viscous contact wave constructed in (11) and (9) with $\left(v_{ \pm}, u_{ \pm}, \theta_{ \pm}\right)$replaced by $\left(v_{ \pm}^{m}, 0, \theta_{ \pm}^{m}\right)$, respectively. We define

$$
\left(\begin{array}{l}
V \\
U \\
\Theta
\end{array}\right)(t, x)=\left(\begin{array}{c}
V_{-}^{r}+V^{c}+V_{+}^{r} \\
U_{-}^{r}+U^{c}+U_{+}^{r} \\
\Theta_{-}^{r}+\Theta^{c}+\Theta_{+}^{r}
\end{array}\right)(t, x)-\left(\begin{array}{c}
v_{-}^{m}+v_{+}^{m} \\
2 u^{m} \\
\theta_{-}^{m}+\theta_{+}^{m}
\end{array}\right),
$$

and

$$
(\phi, \psi, \zeta, \omega)(x, t)=(v-V, u-U, \theta-\Theta, \omega)(t, x) .
$$

Then our main result of this paper is as follows:

Theorem 1.2. For any given $\left(v_{-}, u_{-}, \theta_{-}\right)$, suppose that (16) holds for some small $\delta_{1}>0$. Let $(V, U, \Theta)(t, x)$ be as in (20) with strength $\delta=\left|\theta_{+}-\theta_{-}\right| \leq \tilde{\delta}$. Then there exist positive constants $\delta_{0}\left(\leq \min \left\{\delta_{1}, \tilde{\delta}\right\}\right)$ and $\varepsilon_{0}$, such that if $\delta<\delta_{0}$ and the initial data $\left(v_{0}, u_{0}, \theta_{0}, \omega_{0}\right)$ satisfies

$$
\left\|\left(v_{0}(\cdot)-V(0, \cdot), u_{0}(\cdot)-U(0, \cdot), \theta_{0}(\cdot)-\Theta(0, \cdot), \omega_{0}(\cdot)\right)\right\|_{H^{1}(\mathbb{R})} \leq \varepsilon_{0},
$$

then the Cauchy problem (1)-(2) admits a unique global solution $(v, u, \theta, \omega)(t, x)$ satisfying $(v-V, u-U, \theta-\Theta, \omega)(t, x) \in X([0, \infty))$ and

$$
\lim _{t \rightarrow+\infty} \sup _{x \in \mathbb{R}}\left(\begin{array}{c}
\left|\left(v-v_{-}^{r}-V^{c}-v_{+}^{r}+v_{-}^{m}+v_{+}^{m}\right)(t, x)\right| \\
\left|\left(u-u_{-}^{r}-u_{+}^{r}+u^{m}\right)(t, x)\right| \\
\left|\left(\theta-\theta_{-}^{r}-\Theta^{c}-\theta_{+}^{r}+\theta_{-}^{m}+\theta_{+}^{m}\right)(t, x)\right| \\
|\omega(t, x)|
\end{array}\right)=0 .
$$

Now, we briefly recall some related work in this aspect and make some comments on the analysis in this paper. The nonlinear stability of some basic wave patterns has been studied by many authors. The stability toward contact waves for solutions of systems of viscous conservation laws was first studied by Xin [8] who proved the nonlinear stability of a weak contact discontinuity for the compressible Euler equations with uniform viscosity. Later, Liu and Xin [9] showed the stability of contact discontinuities for a class of general systems of nonlinear conservation laws with uniform viscosity. And this result was improved by Xin 
and Zeng in [10]. The large-time asymptotic nonlinear stability of the supposition of viscous shock waves and contact discontinuities for system of viscous conservation laws with artificial viscosity under small initial perturbations was proved by Zeng [11]. Some interesting results have been obtained for compressible Navier-Stokes system. The asymptotics toward the rarefaction waves for compressible Navier-Stokes system is established in [7] [12] [13] [14]. For a free-boundary value problem, the asymptotic stability of a viscous contact wave of the one-dimensional compressible Navier-Stokes system was first proved by an elementary energy method by Huang, Matsumura, and Shi [15], where the initial perturbation and the strength of the contact discontinuity are suitably small. The asymptotic stability of the linear combination wave of viscous contact wave and the rarefaction waves for the Cauchy problem of the one-dimensional compressible Navier-Stokes system was obtained by Huang, Li and Matsumura in [16], and provided the strength of the combination wave is suitably small. The viscous shock profiles and viscous rarefaction waves have been shown to be asymptotically stable for quite general perturbation for the compressible Navier-Stokes system and more general systems of viscous strictly hyperbolic conservation laws [4] [12] [14] [17]-[22]. There are many results have been obtained for the nonlinear stability of some basic wave patterns consisting of viscous shock waves, rarefaction waves, viscous contact discontinuities and their certain linear superpositions with small perturbation. We refer to [23]-[31] and the references therein for viscous shock waves, [13] [14] [20] for rarefaction waves, [2] [32] [33] [34] [35] for viscous contact discontinuities, [16] [36] [37] [38] for the composition of a viscous contact wave and rarefaction waves. For the corresponding results with large initial perturbation, see [39] [40] [41] and the references cited therein.

The compressible micropolar fluid model has become an important area of interest for mathematicians in the last several decades. The model for compressible flow of micropolar fluid in the one-dimensional case was first studied by N. Mujaković. She considered the local-in-time existence and uniqueness [42], the global existence [43] and regularity of solutions [44] to an initial-boundary value problem with homogeneous boundary conditions of the compressible one-dimensional micropolar fluid system respectively. Other results were proved in [45] [46] [47] for the corresponding non-homogeneous boundary value problems. Besides, she also analyzed large time behavior of the solutions and the stabilization of solutions to the Cauchy problem [48] of the one-dimensional model. There are other authors in [5] [49] showed the nolinear stability of some basic waves (such as rarefaction waves and viscous contact wave etc.). The stability of composite wave for one-dimensional compressible micropolar fluid model without viscosity was studied by Zheng, Chen and Zhang [50]. For the three-dimensional compressible micropolar fluid model, I. Dražić and N. Mujaković in [51] [52] [53] [54] [55] studied the local existence, global existence, uniqueness, large time behavior and regularity of spherical symmetry solutions. 
In this paper, we shall show the asymptotic stability of combination of contact discontinuity with rarefaction waves for the Cauchy problem of the one-dimensional viscous micropolar fluid model, provided the strength of the combination wave is suitably small. After stating some notations, we will reformulate the original problem and give some preliminary lemmas and a priori estimates of solutions to the Cauchy problem (22) in Section 2. Finally, section 3 completes the proof of Theorem 1.2.

Notation. Throughout this paper, several positive generic constants are denoted by $C, c$ without confusion. For functional spaces, $H^{l}(\mathbb{R})$ denotes the th order Sobolev space with its norm

$$
\|f\|_{l}=\sum_{j=0}^{l}\left\|\partial_{x}^{j} f\right\|, \quad \text { where }\|\cdot\| \triangleq\|\cdot\|_{L^{2}(\mathbb{R})} .
$$

\section{Reformation of the Problem and Preliminaries}

Noticing that $\left(V_{ \pm}^{r}, U_{ \pm}^{r}, \Theta_{ \pm}^{r}\right)$ satisfies Euler system (3) and $\left(V^{c}, U^{c}, \Theta^{c}\right)$ satisfies $(1)_{1}$ and (8). To make it more convenient to prove Theorem 1.2, in this section, we will reformulate the problem (1), then the system (1)-(2) be rewritten as

$$
\left\{\begin{array}{l}
\phi_{t}-\psi_{x}=0, \\
\psi_{t}+\left(\frac{R \zeta-P \phi}{v}\right)_{x}=\mu\left(\frac{\psi_{x}}{v}\right)_{x}+F, \\
\frac{R}{\gamma-1} \zeta_{t}+p u_{x}-P U_{x}=\kappa\left(\frac{V \zeta_{x}-\phi \Theta_{x}}{v V}\right)_{x}+\frac{\omega_{x}^{2}}{v}+v \omega^{2}+G, \\
\omega_{t}=A\left[\left(\frac{\omega_{x}}{v}\right)_{x}-v \omega\right] \\
(\phi, \psi, \zeta, \omega)( \pm \infty, t)=0, \\
(\phi, \psi, \zeta, \omega)(x, 0)=\left(\phi_{0}, \psi_{0}, \zeta_{0}, \omega_{0}\right),
\end{array}\right.
$$

where

$$
\begin{aligned}
& P=\frac{R \Theta}{V}, \quad P_{ \pm}=\frac{R \Theta_{ \pm}^{r}}{V_{ \pm}^{r}}, \\
& F=\left[\left(P_{-}+P_{+}-P\right)_{x}\right]+\left[\mu\left(\frac{U_{x}}{v}\right)_{x}-U_{t}^{c}\right] \triangleq F_{1}+F_{2},
\end{aligned}
$$

and

$$
\begin{aligned}
G= & {\left[\left(p^{m}-P\right) U_{x}^{c}+\left(P_{-}-P\right)\left(U_{-}^{r}\right)_{x}+\left(P_{+}-P\right)\left(U_{+}^{r}\right)_{x}\right] } \\
& +\kappa\left[\left(\frac{\Theta_{x}}{V}\right)_{x}-\left(\frac{\Theta_{x}^{c}}{V^{c}}\right)_{x}\right]+\mu \frac{\left(U_{x}+\psi_{x}\right)^{2}}{v} \\
\triangleq & G_{1}+G_{2}+G_{3} .
\end{aligned}
$$

We derive an elementary inequality concerning the heat kernel which will play an essential role later. For $\alpha>0$, we define 


$$
w(t, x)=(1+t)^{-\frac{1}{2}} \exp \left\{-\frac{\alpha x^{2}}{1+t}\right\}, \quad g(t, x)=\int_{-\infty}^{x} w(t, y) \mathrm{d} y
$$

It is easy to check that

$$
4 \alpha g_{t}=w_{x}, \quad\|g(t, \cdot)\|_{L^{\infty}} \leq C \alpha^{-1 / 2} .
$$

Then we have

Lemma 2.1 (see [16]) For $0<T \leq \infty$, suppose that $h(t, x)$ satisfies

$$
h \in L^{\infty}\left(0, T ; L^{2}(\mathbb{R})\right), h_{x} \in L^{2}\left(0, T ; L^{2}(\mathbb{R})\right), h_{t} \in L^{2}\left(0, T ; H^{-1}(\mathbb{R})\right) .
$$

Then the following estimate holds:

$$
\int_{0}^{T} \int_{\mathbb{R}} h^{2} w^{2} \mathrm{~d} x \mathrm{~d} t \leq 4 C\|h(x, 0)\|^{2}+4 C \alpha^{-1} \int_{0}^{T}\left\|h_{x}(t)\right\|^{2} \mathrm{~d} t+8 \alpha \int_{0}^{T}\left\langle h_{t}, h g^{2}\right\rangle_{H^{-1} \times H^{1}} \mathrm{~d} t
$$

For the proof Lemma 2.1, one refers to [16]. Next, we summarize some basic properties of the viscous contact wave $\left(V^{c}, U^{c}, \Theta^{c}\right)$.

Lemma 2.2 (see [36]) Assume that $\delta=\left|\theta_{+}-\theta_{-}\right| \leq \tilde{\delta}$ for some positive constant $\tilde{\delta}$. Then there exists two positive constants $c_{0}$ and $C$ such that the viscous contact wave $\left(V^{c}, U^{c}, \Theta^{c}\right)$ satisfies the following estimates:

$$
\begin{gathered}
\left|V^{c}-v_{ \pm}\right|+\left|\Theta^{c}-\theta_{ \pm}\right| \leq C \delta \mathrm{e}^{-\frac{c_{0} x^{2}}{1+t}}, \quad\left|U^{c}-u_{-}\right| \leq C \delta(1+t)^{-\frac{1}{2}} \mathrm{e}^{-\frac{c_{0} x^{2}}{1+t}}, \\
\left|\partial_{x}^{k} V^{c}\right|+\left|\partial_{x}^{k} \Theta^{c}\right| \leq C \delta(1+t)^{-\frac{k}{2}} \mathrm{e}^{-\frac{c_{0} x^{2}}{1+t}}, \quad\left|\partial_{x}^{l} U^{c}\right| \leq C \delta(1+t)^{-\frac{l+1}{2}} \mathrm{e}^{-\frac{c_{0} x^{2}}{1+t}}, \quad k, l \in Z^{+} .
\end{gathered}
$$

Lemma 2.2 can be proved directly from Equations (10) and (11), the details are omitted here. The solution $\hat{\omega}(t, x)$ of the Cauchy problem (19) has the following properties.

Lemma 2.3 (see [7]) For given $\hat{\omega}_{l} \in \mathbb{R}$, and $\bar{\omega}>0$, let $\hat{\omega}_{r} \in\left\{\hat{\omega} \mid 0<\tilde{\omega} \triangleq \hat{\omega}-\hat{\omega}_{l}<\bar{\omega}\right\}$. Then the problem (19) has a unique smooth global solution in time satisfying the following.

i) $\hat{\omega}_{l}<\hat{\omega}(t, x)<\hat{\omega}_{r}, \hat{\omega}_{x}>0, x \in \mathbb{R}, t>0$.

ii) For any $p \in[1,+\infty]$, there exists some positive constant $C=C\left(p, \hat{\omega}_{l}, \bar{\omega}\right)$ such that for $\tilde{\omega} \geq 0$ and $t \geq 0$,

$$
\left\|\hat{\omega}_{x}(t)\right\|_{L^{p}} \leq C \min \left\{\tilde{\omega}, \tilde{\omega}^{\frac{1}{p}}(1+t)^{-1+\frac{1}{p}}\right\}, \quad\left\|\hat{\omega}_{x x}(t)\right\|_{L^{p}} \leq C \min \left\{\tilde{\omega},(1+t)^{-1}\right\} .
$$

iii) If $\hat{\omega}_{l}>0$, for any $(t, x) \in[0,+\infty) \times(-\infty, 0]$

$$
\left|\hat{\omega}(t, x)-\hat{\omega}_{l}\right| \leq \tilde{\omega} \mathrm{e}^{-2\left(|x|+\hat{\omega}_{l} t\right)}, \quad\left|\hat{\omega}_{x}(t, x)\right| \leq 2 \tilde{\omega} \mathrm{e}^{-2\left(|x|+\hat{\omega}_{l} t\right)} .
$$

iv) If $\hat{\omega}_{r}<0$, for any $(t, x) \in[0,+\infty) \times[0,+\infty)$,

$$
\left|\hat{\omega}(t, x)-\hat{\omega}_{r}\right| \leq \tilde{\omega} \mathrm{e}^{-2\left(|x|+\left|\hat{\omega}_{r}\right| t\right)}, \quad\left|\hat{\omega}_{x}(t, x)\right| \leq 2 \tilde{\omega} \mathrm{e}^{-2\left(|x|+\left|\hat{\omega}_{r}\right| t\right)} .
$$

v) For the Riemann solution $\omega^{r}\left(\frac{x}{t}\right)$ of the scalar Equation (19) $)_{1}$ with the Riemann initial data 


$$
\hat{\omega}(0, x)= \begin{cases}\hat{\omega}_{l}, & x<0 \\ \hat{\omega}_{r}, & x>0,\end{cases}
$$

we have

$$
\limsup _{t \rightarrow+\infty}\left|\hat{\omega}(t, x)-\omega^{r}\left(\frac{x}{t}\right)\right|=0 .
$$

We use Lemma 2.3 to investigate the properties of the smooth rarefaction waves $\left(V_{ \pm}^{r}, U_{ \pm}^{r}, \Theta_{ \pm}^{r}\right)$ constructed in (20) and the viscous contact wave $\left(V^{c}, U^{c}, \Theta^{c}\right)(t, x)$, we divided the domain $\mathbb{R} \times(0, t)$ into three parts, that is, $\mathbb{R} \times(0, t)=\Omega_{-} \cup \Omega_{c} \cup \Omega_{+}$with

$$
\Omega_{ \pm}=\left\{(x, t) \mid \pm 2 x> \pm \lambda_{ \pm}\left(v_{ \pm}^{m}, s_{ \pm}\right) t\right\},
$$

and

$$
\Omega_{c}=\left\{(x, t) \mid \lambda_{-}\left(v_{-}^{m}, s_{-}\right) t \leq 2 x \leq \lambda_{+}\left(v_{+}^{m}, s_{+}\right) t\right\} .
$$

Then, Lemma 2.3 and (10) easily give

Lemma 2.4. (see [16]) For any given $\left(v_{-}, u_{-}, \theta_{-}\right)$, assume that $\left(v_{+}, u_{+}, \theta_{+}\right)$ satisfies (16) with $\delta=\left|\theta_{-}-\theta_{+}\right| \leq \tilde{\delta}$. Then the smooth rarefaction waves $\left(V_{ \pm}^{r}, U_{ \pm}^{r}, \Theta_{ \pm}^{r}\right)$ constructed in (18) and the viscous contact discontinuity wave $\left(V^{c}, U^{c}, \Theta^{c}\right)$ satisfy the following.

i) $\left(U_{ \pm}^{r}\right)_{x} \geq 0, x \in \mathbb{R}, t>0$.

ii) For any $p \in[1,+\infty]$, there exists some positive constant $C=C\left(p, v_{-}, u_{-}, \theta_{-}, \delta_{1}, \tilde{\delta}\right)$ such that for $\delta=\left|\theta_{-}-\theta_{+}\right|$and $t \geq 0$,

$$
\begin{aligned}
& \left\|\left(\left(V_{ \pm}^{r}\right)_{x},\left(U_{ \pm}^{r}\right)_{x},\left(\Theta_{ \pm}^{r}\right)_{x}\right)(t)\right\|_{L^{p}} \leq C \min \left\{\delta, \delta^{\frac{1}{p}} t^{-1+\frac{1}{p}}\right\}, \\
& \left\|\left(\partial_{x}^{k} V_{ \pm}^{r}, \partial_{x}^{k} U_{ \pm}^{r}, \partial_{x}^{k} \Theta_{ \pm}^{r}\right)(t)\right\|_{L^{p}} \leq C \min \left\{\delta, t^{-1}\right\}, \quad k=2,3 .
\end{aligned}
$$

iii) There exists some positive constant $C=C\left(v_{-}, u_{-}, \theta_{-}, \delta_{1}, \tilde{\delta}\right)$ such that for $\delta=\left|\theta_{-}-\theta_{+}\right|$and

$$
c_{0}=\frac{1}{10} \min \left\{\left|\lambda_{-}\left(v_{-}^{m}, s_{-}\right)\right|, \lambda_{+}\left(v_{+}^{m}, s_{+}\right), c_{2} \lambda_{-}^{2}\left(v_{-}^{m}, s_{-}\right), c_{2} \lambda_{+}^{2}\left(v_{+}^{m}, s_{+}\right), 1\right\},
$$

we have in $\Omega_{c}$ that

$$
\left|\left(\left(V_{ \pm}^{r}\right)_{x},\left(U_{ \pm}^{r}\right)_{x},\left(\Theta_{ \pm}^{r}\right)_{x}\right)\right|+\left|V_{ \pm}^{r}-v_{ \pm}^{m}\right|+\left|\Theta_{ \pm}^{r}-\theta_{ \pm}^{m}\right| \leq C \delta \mathrm{e}^{-c_{0}(|x|+t)},
$$

and in $\Omega_{\mp}$,

$$
\begin{aligned}
& \left|V_{x}^{c}\right|+\left|\Theta_{x}^{c}\right|+\left|V^{c}-v_{ \pm}^{m}\right|+\left|\Theta^{c}-\theta_{ \pm}^{m}\right|+\left|U_{x}^{c}\right| \leq C \delta \mathrm{e}^{-c_{0} \mid(x \mid+t),} \\
& \left|\left(\left(V_{ \pm}^{r}\right)_{x},\left(U_{ \pm}^{r}\right)_{x},\left(\Theta_{ \pm}^{r}\right)_{x}\right)\right|+\left|V_{ \pm}^{r}-v_{ \pm}^{m}\right|+\left|\Theta_{ \pm}^{r}-\theta_{ \pm}^{m}\right| \leq C \delta \mathrm{e}^{-c_{0}(|x|+t)} .
\end{aligned}
$$

iv) For the rarefaction waves $\left(v_{ \pm}^{r}, u_{ \pm}^{r}, \theta_{ \pm}^{r}\right)\left(\frac{x}{t}\right)$ determined by (3) and (17), it holds

$$
\lim _{t \rightarrow+\infty} \sup _{x \in \mathbb{R}}\left|\left(V_{ \pm}^{r}, U_{ \pm}^{r}, \Theta_{ \pm}^{r}\right)(t, x)-\left(v_{ \pm}^{r}, u_{ \pm}^{r}, \theta_{ \pm}^{r}\right)\left(\frac{x}{t}\right)\right|=0 .
$$


Finally, we give a Sobolev inequality without proof.

Lemma 2.5. (see [32]) For any $f(x) \in H^{1}(\mathbb{R})$, we have

$$
\|f\|_{L^{\infty}} \leq\|f\|^{\frac{1}{2}}\left\|f_{x}\right\|^{\frac{1}{2}} .
$$

Since the local existence of the solution is well known (for example, see [42]), to prove the global existence part of Theorems 1.2, we only have to establish the following a priori estimates.

\section{Proposition 2.1}

(A priori estimate) There exist positive constants $\varepsilon_{0} \leq 1, \delta_{0} \leq \min \left\{\delta_{1}, \tilde{\delta}, 1\right\}$ and $C$, such that for $T>0$ and $(\phi, \psi, \zeta, \omega) \in X([0, T])$ satisfying

$$
N(T)=\sup _{0 \leq t \leq T}\|(\phi, \psi, \zeta, \omega)(t)\|_{1}^{2}<\varepsilon_{0}, \quad\left|\theta_{-}-\theta_{+}\right|=\delta<\delta_{0},
$$

it follows the estimate

$$
\begin{aligned}
& \sup _{0 \leq t \leq T}\|(\phi, \psi, \zeta, \omega)(t)\|_{1}^{2}+\int_{0}^{T}\left(\left\|\phi_{x}(s)\right\|^{2}+\left\|\left(\psi_{x}, \zeta_{x}\right)(s)\right\|_{1}^{2}+\|\omega(s)\|_{2}^{2}\right) \mathrm{d} s \\
& \leq C\left(\delta^{\frac{1}{6}}+\left\|\left(\phi_{0}, \psi_{0}, \zeta_{0}, \omega_{0}\right)\right\|_{1}^{2}\right) .
\end{aligned}
$$

Once Proposition 2.1 is proved, we can extend the unique local solution $(v, u, \theta, \omega)$ which can be obtained as in [42], to $T=\infty$. Estimate (32) together with the Equation (22) implies that

$$
\int_{0}^{\infty}\left(\left\|\left(\phi_{x}, \psi_{x}, \zeta_{x}, \omega_{x}\right)(t)\right\|^{2}+\left|\frac{\mathrm{d}}{\mathrm{d} t}\left\|\left(\phi_{x}, \psi_{x}, \zeta_{x}, \omega_{x}\right)(t)\right\|^{2}\right|\right) \mathrm{d} t<\infty,
$$

which as well as (32) and the Sobolev inequality easily leads to the asymptotic behavior of the solutions, that is, (21).

From now on until the end of this paper, we always assume that $\varepsilon_{0}+\delta_{0} \leq 1$. Proposition 2.1 is an easy consequence of the following lemmas.

\section{Energy Estimates}

In this section we will drive some a priori energy estimates for the solutions to the system (1). Since Theorem 1.1 has been proved by Liu and Yin that we can see the details in [5], we will give here the proof of Theorem 1.2 for brevity. We first give the following key estimate.

Lemma 3.1. For $T>0$ and $(\phi, \psi, \zeta, \omega) \in X([0, T])$ satisfying (31) with suitably small $\varepsilon_{0}+\delta_{0}$, we have for $t \in[0, T]$,

$$
\begin{aligned}
& \|(\phi, \psi, \zeta, \omega)(t)\|^{2}+\int_{0}^{t}\left\|\left(\psi_{x}, \zeta_{x}, \omega_{x}, \omega\right)(s)\right\|^{2} \mathrm{~d} s \\
& +\int_{0}^{t} \int_{\mathbb{R}}\left(\phi^{2}+\zeta^{2}\right)\left(\left(U_{-}^{r}\right)_{x}+\left(U_{+}^{r}\right)_{x}\right) \mathrm{d} x \mathrm{~d} s \\
& \leq C\left(\delta^{\frac{1}{6}}+\left\|\left(\phi_{0}, \psi_{0}, \zeta_{0}, \omega_{0}\right)\right\|^{2}+\delta^{\frac{1}{2}} \int_{0}^{t}\left\|\phi_{x}(s)\right\|^{2} \mathrm{~d} s\right) .
\end{aligned}
$$

Proof. First, we use Lemma 2.4 to investigate some aspects of $F$ and $G$.

Since $R \Theta^{c}=p^{m} V^{c}$ direct calculation yields that 


$$
\begin{aligned}
\frac{F_{1}}{R}= & \left(\frac{\Theta_{-}^{r}}{V_{-}^{r}}+\frac{\Theta_{+}^{r}}{V_{+}^{r}}+\frac{\Theta^{c}}{V^{c}}-\frac{\Theta}{V}\right)_{x} \\
= & \left(\frac{\Theta_{-}^{r}}{V_{-}^{r}}+\frac{\Theta_{+}^{r}}{V_{+}^{r}}+\frac{\Theta^{c}}{V^{c}}-\frac{\Theta_{-}^{r}+\Theta_{+}^{r}+\Theta^{c}-\Theta_{-}^{m}-\Theta_{+}^{m}}{V}\right)_{x} \\
= & \left(\Theta_{-}^{r}\right)_{x}\left(\frac{1}{V_{-}^{r}}-\frac{1}{V}\right)+\left(\Theta_{+}^{r}\right)_{x}\left(\frac{1}{V_{+}^{r}}-\frac{1}{V}\right)+\Theta_{x}^{c}\left(\frac{1}{V^{c}}-\frac{1}{V}\right) \\
& +\left(V_{-}^{r}\right)_{x}\left(\frac{\Theta}{V^{2}}-\frac{\Theta_{-}^{r}}{\left(V_{-}^{r}\right)^{2}}\right)+\left(V_{+}^{r}\right)_{x}\left(\frac{\Theta}{V^{2}}-\frac{\Theta_{+}^{r}}{\left(V_{+}^{r}\right)^{2}}\right)+V_{x}^{c}\left(\frac{\Theta}{V^{2}}-\frac{\Theta^{c}}{\left(V^{c}\right)^{2}}\right) .
\end{aligned}
$$

It follows from (20) that

$$
\left|\left(\Theta_{-}^{r}\right)_{x}\left(\left(V_{-}^{r}\right)^{-1}-V^{-1}\right)\right| \leq C\left|\left(\Theta_{-}^{r}\right)_{x}\right|\left(\left|V_{+}^{r}-v_{+}^{m}\right|+\left|V^{c}-v_{-}^{m}\right|\right),
$$

thus, it derives from Lemma 2.4 that

$$
\begin{aligned}
& \left|\left(\Theta_{-}^{r}\right)_{x}\left(\left(V_{-}^{r}\right)^{-1}-V^{-1}\right)\right| \\
& \leq\left. C\left|\left(\Theta_{-}^{r}\right)_{x}\right|\left(\left|V_{+}^{r}-v_{+}^{m}\right|+\left|V^{c}-v_{-}^{m}\right|\right)\right|_{\Omega_{-}}+\left.C\left|\left(\Theta_{-}^{r}\right)_{x}\right|\left(\left|V_{+}^{r}-v_{+}^{m}\right|+\left|V^{c}-v_{-}^{m}\right|\right)\right|_{\Omega_{+} \cup \Omega_{c}} \\
& \leq\left. C\left(\left|V_{+}^{r}-v_{+}^{m}\right|+\left|V^{c}-v_{-}^{m}\right|\right)\right|_{\Omega_{-}}+C\left|\left(\Theta_{-}^{r}\right)_{x}\right| \Omega_{+} \cup \Omega_{c} \\
& \leq C \delta \mathrm{e}^{-c_{0}(|x|+t)} .
\end{aligned}
$$

We can treat the other terms on the righthand side of (34) in the same way to obtain

$$
\left|F_{1}\right| \leq C \delta \mathrm{e}^{-c_{0}(|x|+t)} .
$$

Since

$$
\begin{aligned}
\left|F_{2}\right| \leq & C\left(\left|U_{t}^{c}\right|+\left|\left(U_{-}^{r}\right)_{x x}\right|+\left|\left(U_{+}^{r}\right)_{x x}\right|+\left|U_{x x}^{c}\right|\right. \\
& \left.+\left(U_{-}^{r}\right)_{x}\left|\left(V_{-}^{r}\right)_{x}\right|+\left(U_{+}^{r}\right)_{x}\left|\left(V_{+}^{r}\right)_{x}\right|+\left|U_{x}^{c}\right|\left|V_{x}^{c}\right|\right) \\
& +C\left[\left(U_{-}^{r}\right)_{x}\left(\left|\left(V_{+}^{r}\right)_{x}\right|+\left|V_{x}^{c}\right|\right)+\left(U_{+}^{r}\right)_{x}\left(\left|\left(V_{-}^{r}\right)_{x}\right|+\left|V_{x}^{c}\right|\right)\right. \\
& \left.+\left|U_{x}^{c}\right|\left(\left|\left(V_{-}^{r}\right)_{x}\right|+\left|\left(V_{+}^{r}\right)_{x}\right|\right)\right]+C\left|\phi_{x}\right|\left(\left(U_{-}^{r}\right)_{x}+\left(U_{+}^{r}\right)_{x}+\left|U_{x}^{c}\right|\right) \\
= & F_{2}^{1}+F_{2}^{2}+F_{2}^{3},
\end{aligned}
$$

the estimate (10) and Lemma 2.4 imply

$$
\begin{gathered}
\left\|F_{2}^{1}\right\|_{L^{1}} \leq C \delta^{\frac{1}{8}}(1+t)^{-\frac{7}{8}}, \\
\left\|F_{2}^{2}\right\|_{L^{1}} \leq C \delta \mathrm{e}^{-c_{0} t},
\end{gathered}
$$

and

$$
\left\|F_{2}^{3}\right\|_{L^{1}} \leq C \delta^{\frac{1}{2}}(1+t)^{-\frac{1}{2}}\left\|\phi_{x}\right\| \leq C \delta^{\frac{1}{2}}(1+t)^{-1}+C \delta^{\frac{1}{2}}\left\|\phi_{x}\right\|^{2} .
$$

The estimates (36)-(40) give 


$$
\|F\|_{L^{1}} \leq C \delta^{\frac{1}{8}}(1+t)^{-\frac{7}{8}}+C \delta^{\frac{1}{2}}\left\|\phi_{x}\right\|^{2} .
$$

Similar to (36), we have

$$
\left|G_{1}\right| \leq C \delta \mathrm{e}^{-c_{0}(|x|+t)} .
$$

Since

$$
\begin{aligned}
G_{2} & =\kappa\left(V^{-1}\left(\left(\Theta_{-}^{r}\right)_{x}+\left(\Theta_{+}^{r}\right)_{x}\right)\right)_{x}+\kappa\left(\Theta_{x}^{c}\left(V^{-1}-\left(V^{c}\right)^{-1}\right)\right)_{x} \\
& =G_{2}^{1}+G_{2}^{2},
\end{aligned}
$$

and

$$
\begin{aligned}
\left|G_{2}^{1}\right| \leq & C\left(\left|\left(\Theta_{-}^{r}\right)_{x x}\right|+\left|\left(\Theta_{+}^{r}\right)_{x x}\right|+\left|\left(\Theta_{-}^{r}\right)_{x}\right|\left|\left(V_{-}^{r}\right)_{x}\right|+\left|\left(\Theta_{+}^{r}\right)_{x}\right|\left|\left(V_{+}^{r}\right)_{x}\right|\right) \\
& \left.+C\left|\left(\Theta_{-}^{r}\right)_{x}\right|\left|\left(\mid V_{+}^{r}\right)_{x}\right|+\left|V_{x}^{c}\right|\right)+C\left|\left(\Theta_{+}^{r}\right)_{x}\right|\left(\left|\left(V_{-}^{r}\right)_{x}\right|+\left|V_{x}^{c}\right|\right) .
\end{aligned}
$$

It follows from (10) and Lemma 2.4 that

$$
\begin{aligned}
& \left\|G_{2}^{1}\right\|_{L^{1}} \leq C \delta^{\frac{1}{8}}(1+t)^{-\frac{7}{8}}, \\
& \left|G_{2}^{2}\right| \leq C\left(\left|\Theta_{x x}^{c}\right|+\left|\Theta_{x}^{c}\right|\left|V_{x}^{c}\right|\right)\left(\left|V_{+}^{r}-v_{+}^{m}\right|+\left|V_{-}^{r}-v_{-}^{m}\right|\right)+C\left|\Theta_{x}^{c}\right|\left(\left|\left(V_{-}^{r}\right)_{x}\right|+\left|\left(V_{+}^{r}\right)_{x}\right|\right) \\
& \leq C \delta \mathrm{e}^{-c_{0}(|x|+t)},
\end{aligned}
$$

and

$$
\begin{aligned}
\left\|G_{3}\right\|_{L^{1}} & \leq C\left\|\left(U_{-}^{r}\right)_{x}\right\|^{2}+C\left\|\left(U_{+}^{r}\right)_{x}\right\|^{2}+C\left\|U_{x}^{c}\right\|^{2}+C\left\|\psi_{x}\right\|^{2} \\
& \leq C \delta(1+t)^{-1}+C\left\|\psi_{x}\right\|^{2} .
\end{aligned}
$$

Thus, one derives from (34), (42)-(47) that

$$
\|G\|_{L^{1}} \leq C \delta^{\frac{1}{8}}(1+t)^{-\frac{7}{8}}+C\left\|\psi_{x}\right\|^{2} .
$$

Now, multiplying $(22)_{1}$ by $-R \Theta\left(v^{-1}-V^{-1}\right),(22)_{2}$ by $\psi$ and $(22)_{3}$ by $\zeta \theta^{-1}$, $(22)_{4}$ by $\omega$, then adding the resulting equations together, and using that

$$
\begin{gathered}
R \Theta\left(\frac{1}{v}-\frac{1}{V}\right) \phi_{t}+\left[R \Theta \Phi\left(\frac{v}{V}\right)\right]_{t}=-\frac{P V_{t}}{v V} \phi^{2}+R \Theta_{t} \Phi\left(\frac{v}{V}\right), \\
{\left[\Theta \Phi\left(\frac{\theta}{\Theta}\right)\right]_{t}=\frac{\zeta}{\theta} \zeta_{t}-\Phi\left(\frac{\Theta}{\theta}\right) \Theta_{t},}
\end{gathered}
$$

then we have

$$
\begin{aligned}
& \left\{R \Theta \Phi\left(\frac{v}{V}\right)+\frac{1}{2} \psi^{2}+\frac{R}{\gamma-1} \Theta \Phi\left(\frac{\theta}{\Theta}\right)+\frac{\omega^{2}}{2}\right\}_{t}+\mu \frac{\psi_{x}^{2}}{v}+\frac{\kappa \zeta_{x}^{2}}{v \theta} \\
& +A v \omega^{2}+\frac{A}{v} \omega_{x}^{2}+H_{1 x}+Q_{1}+Q_{2}=F \psi+G \frac{\zeta}{\theta}+Q_{3},
\end{aligned}
$$

where 


$$
\begin{aligned}
& H_{1}=(p-P) \psi-\mu \frac{\psi \psi_{x}}{v}-\frac{\kappa \zeta}{\theta}\left(\frac{\zeta_{x}}{v}-\frac{\Theta_{x} \phi}{v V}\right)-\frac{A \omega \omega_{x}}{v}, \\
& Q_{1}=-R \Theta_{t} \Phi\left(\frac{v}{V}\right)+\frac{P U_{x}}{v V} \phi^{2}+\frac{R \Theta_{t}}{\gamma-1} \Phi\left(\frac{\Theta}{\theta}\right)+\frac{\zeta}{\theta}(p-P) U_{x}, \\
& Q_{2}=-\kappa \frac{\theta_{x}}{\theta^{2} v} \zeta \zeta_{x}-\kappa \frac{\zeta_{x} \phi}{\theta v V} \Theta_{x}+\kappa \frac{\theta_{x} \zeta \phi}{\theta^{2} v V} \Theta_{x}, \\
& Q_{3}=\frac{\zeta}{\theta}\left(\frac{\omega_{x}^{2}}{v}+v \omega^{2}\right) \\
& \Phi(s)=s-1-\ln s .
\end{aligned}
$$

Notice that $\Phi(1)=\Phi^{\prime}(1)=0$ and $\Phi^{\prime \prime}(s)>0$, there exists positive constants $C_{1}$ and $C_{2}$ such that

$$
C_{1} \phi^{2} \leq \Phi\left(\frac{v}{V}\right), \Phi\left(\frac{V}{v}\right) \leq C_{2} \phi \text {, and } C_{1} \zeta^{2} \leq \Phi\left(\frac{\theta}{\Theta}\right), \Phi\left(\frac{\Theta}{\theta}\right) \leq C_{2} \zeta^{2} .
$$

Noticing that

$$
\begin{aligned}
-R \Theta_{t}= & (\gamma-1) P_{-}\left(U_{-}^{r}\right)_{x}+(\gamma-1) P_{+}\left(U_{+}^{r}\right)_{x}-p^{m} U_{x}^{c} \\
= & (\gamma-1) P\left(\left(U_{-}^{r}\right)_{x}+\left(U_{+}^{r}\right)_{x}\right)+(\gamma-1)\left(P_{-}-P\right)\left(U_{-}^{r}\right)_{x} \\
& +(\gamma-1)\left(P_{+}-P\right)\left(U_{+}^{r}\right)_{x}-p^{m} U_{x}^{c},
\end{aligned}
$$

we have

$$
\begin{aligned}
Q_{1} & =-R \Theta_{t}\left[\Phi\left(\frac{v}{V}\right)-\frac{1}{\gamma-1} \Phi\left(\frac{\Theta}{\theta}\right)\right]+\left[\frac{P \phi^{2}}{v V}+\frac{\zeta}{\theta}(p-P)\right] U_{x} \\
& =\left(\left(U_{-}^{r}\right)_{x}+\left(U_{+}^{r}\right)_{x}\right) Q_{11}+Q_{12},
\end{aligned}
$$

where

$$
\begin{aligned}
Q_{11} & =(\gamma-1) P(V, \Theta) \Phi\left(\frac{v}{V}\right)+\frac{P \phi^{2}}{v V}-P \Phi\left(\frac{\Theta}{\theta}\right)+\frac{\zeta}{\theta}(p-P) \\
& =P\left(\frac{\theta V}{\Theta v}-1+\gamma\left(\frac{v}{V}-1\right)-\left(\ln \frac{\theta}{\Theta}+(\gamma-1) \ln \frac{v}{V}\right)\right) \\
& =P\left(\Phi\left(\frac{\theta V}{\Theta v}\right)+\gamma \Phi\left(\frac{v}{V}\right)\right) \geq C_{0}\left(\phi^{2}+\zeta^{2}\right),
\end{aligned}
$$

and

$$
\begin{aligned}
Q_{12}= & U_{x}^{c}\left[\frac{P(V, \Theta) \phi^{2}}{v V}-p^{m} \Phi\left(\frac{v}{V}\right)+\frac{p^{m}}{\gamma-1} \Phi\left(\frac{\Theta}{\theta}\right)+\frac{\zeta}{\theta}(p-P)\right] \\
& +(\gamma-1)\left(P_{-}-P\right)\left(U_{-}^{r}\right)_{x}\left(\Phi\left(\frac{v}{V}\right)-\frac{1}{\gamma-1} \Phi\left(\frac{\Theta}{\theta}\right)\right) \\
& +(\gamma-1)\left(P_{+}-P\right)\left(U_{+}^{r}\right)_{x}\left(\Phi\left(\frac{v}{V}\right)-\frac{1}{\gamma-1} \Phi\left(\frac{\Theta}{\theta}\right)\right),
\end{aligned}
$$

satisfies

$$
\begin{aligned}
\left|Q_{12}+Q_{2}\right| & \leq C_{\eta}\left(\phi^{2}+\zeta^{2}\right)\left(\Theta_{x}^{2}+\left|\Theta_{x x}^{c}\right|\right)+\eta \zeta_{x}^{2}+C \delta \mathrm{e}^{-c_{0}(|x|+t)} \\
& \leq C_{\eta}\left(\phi^{2}+\zeta^{2}\right)\left(\delta^{\frac{1}{4}}(1+t)^{-\frac{7}{4}}+\delta(1+t)^{-1} \mathrm{e}^{-\frac{c_{0} x^{2}}{1+t}}\right)+\eta \zeta_{x}^{2}+C \delta \mathrm{e}^{-c_{0}(|x|+t),}
\end{aligned}
$$


due to Lemma 2.4 and (10). It is easy to compute

$$
\left|Q_{3}\right| \leq C\|\zeta\|_{L^{\infty}}\left(\omega_{x}^{2}+\omega^{2}\right) \leq C \varepsilon_{0}\left(\omega_{x}^{2}+\omega^{2}\right) .
$$

After integrating (51) on $(0, t) \times \mathbb{R}$, we deduce from (30), (41), (48), (22), (56), (28) and (59) that

$$
\begin{aligned}
& \|(\phi, \psi, \zeta, \omega)(t)\|^{2}+\int_{0}^{t}\left\|\left(\psi_{x}, \zeta_{x}, \omega_{x}, \omega\right)(s)\right\|^{2} \mathrm{~d} s \\
& +\int_{0}^{t} \int_{\mathbb{R}}\left(\phi^{2}+\zeta^{2}\right)\left(\left(U_{-}^{r}\right)_{x}+\left(U_{+}^{r}\right)_{x}\right) \mathrm{d} x \mathrm{~d} s \\
& \leq C\left\|\left(\phi_{0}, \psi_{0}, \zeta_{0}, \omega_{0}\right)\right\|^{2}+C \delta^{\frac{1}{6}}+C \delta^{\frac{1}{2}} \int_{0}^{t}\left\|\phi_{x}(s)\right\|^{2} \mathrm{~d} s \\
& \quad+C \delta \int_{0}^{t}(1+s)^{-1} \int_{\mathbb{R}}\left(\zeta^{2}+\phi^{2}\right) \mathrm{e}^{-\frac{c_{0} x^{2}}{1+t}} \mathrm{~d} x \mathrm{~d} s .
\end{aligned}
$$

Lemma 3.1 thus follows directly from (60) and the following Lemma 3.2 by choosing $\alpha=c_{2} / 4$ in (61) and $\delta$ suitably small.

Lemma 3.2. For $\alpha \in\left(0, c_{2} / 4\right]$ and $w$ defined in (25), there exists some positive constant $C$ depending on $\alpha$ such that the following estimate holds

$$
\begin{aligned}
& \int_{0}^{t} \int_{\mathbb{R}}\left(\phi^{2}+\psi^{2}+\zeta^{2}\right) w^{2} \mathrm{~d} x \mathrm{~d} s \\
& \leq C+C \int_{0}^{t}\left\|\left(\phi_{x}, \psi_{x}, \zeta_{x}, \omega_{x}, \omega\right)\right\|^{2} \mathrm{~d} s+C \int_{0}^{t} \int_{\mathbb{R}}\left(\phi^{2}+\zeta^{2}\right)\left(\left(U_{-}^{r}\right)_{x}+\left(U_{+}^{r}\right)_{x}\right) \mathrm{d} x \mathrm{~d} s .
\end{aligned}
$$

Proof. The proof of (61) is divided into the following two parts:

$$
\begin{aligned}
& \int_{0}^{t} \int_{\mathbb{R}}\left((R \zeta-P \phi)^{2}+\psi^{2}\right) w^{2} \mathrm{~d} x \mathrm{~d} s \\
& \leq C+C \int_{0}^{t}\left\|\left(\phi_{x}, \psi_{x}, \zeta_{x}, \omega_{x}, \omega\right)\right\|^{2} \mathrm{~d} s+C \delta \int_{0}^{t} \int_{\mathbb{R}}\left(\phi^{2}+\zeta^{2}\right) w^{2} \mathrm{~d} x \mathrm{~d} s,
\end{aligned}
$$

and for any $\eta>0$,

$$
\begin{aligned}
& \int_{0}^{t} \int_{\mathbb{R}}(R \zeta+(\gamma-1) P \phi)^{2} w^{2} \mathrm{~d} x \mathrm{~d} s \\
& \leq C+\frac{C}{\eta} \int_{0}^{t}\left\|\left(\phi_{x}, \psi_{x}, \zeta_{x}, \omega_{x}, \omega\right)\right\|^{2} \mathrm{~d} s+C(\delta+\eta) \int_{0}^{t} \int_{\mathbb{R}}\left(\phi^{2}+\zeta^{2}\right) w^{2} \mathrm{~d} x \mathrm{~d} s \\
& \quad+C \int_{0}^{t} \int_{\mathbb{R}}\left(\phi^{2}+\zeta^{2}\right)\left(\left(U_{-}^{r}\right)_{x}+\left(U_{+}^{r}\right)_{x}\right) \mathrm{d} x \mathrm{~d} s .
\end{aligned}
$$

In fact, adding (63) to (62) and taking first $\eta$ then $\delta$ suitably small thus implies (61) easily.

Here we used the same method as in [16] and combined with [50], then, we can complete the proof of Lemma 3.2. We omit the details for simplicity.

Lemma 3.3. Suppose that $(\phi, \psi, \zeta, \omega) \in X([0, T])$ satisfies (31) with suitably small $\varepsilon_{0}+\delta_{0}$. Then it holds for $t \in[0, T]$,

$$
\left\|\phi_{x}(t)\right\|^{2}+\int_{0}^{t}\left\|\phi_{x}(s)\right\|^{2} \mathrm{~d} s \leq C\left(\delta^{\frac{1}{6}}+\left\|\left(\psi_{0}, \zeta_{0}, \omega_{0}\right)\right\|^{2}+\left\|\phi_{0}\right\|_{1}^{2}\right) .
$$

Proof. We rewrite Equation $(22)_{2}$ as

$$
\begin{aligned}
& \mu\left(\frac{\phi_{x}}{v}\right)_{t}+\left(P+\frac{R \zeta-P \phi}{v}\right) \frac{\phi_{x}}{v} \\
& =\psi_{t}+\frac{R \zeta_{x}}{v}-\frac{R \zeta-P \phi}{v^{2}} V_{x}-\mu\left(\frac{V_{x}}{v}\right)_{t}-\frac{\phi P_{x}}{v}+U_{t}^{c}-F_{1},
\end{aligned}
$$


where we have used the following simple fact

$$
\left(\frac{u_{x}}{v}\right)_{x}=\left(\frac{v_{t}}{v}\right)_{x}=\left(\frac{v_{x}}{v}\right)_{t}=\left(\frac{\phi_{x}}{v}\right)_{t}+\left(\frac{V_{x}}{v}\right)_{t},
$$

due to $v_{t}=u_{x}$. Multiplying (65) by $\frac{\phi_{x}}{v}$, using $\phi_{t}=\psi_{x}$, noticing that

$$
\left(\frac{\phi_{x}}{v}\right)_{t}=\frac{\psi_{x x}}{v}-\frac{\phi_{x} \psi_{x}+\phi_{x} U_{x}}{v^{2}}
$$

we have

$$
\begin{aligned}
& \left(\frac{\mu}{2} \frac{\phi_{x}^{2}}{v^{2}}-\psi \frac{\phi_{x}}{v}\right)_{t}+\left(P+\frac{R \zeta-P \phi}{v}\right) \frac{\phi_{x}^{2}}{v^{2}} \\
& =-\left(\frac{\psi \psi_{x}}{v}\right)_{x}+\frac{\psi_{x}^{2}}{v}+\frac{\psi \phi_{x} U_{x}}{v^{2}}-\frac{\psi \psi_{x} V_{x}}{v^{2}}-\frac{R \zeta-P \phi}{v^{3}} V_{x} \phi_{x}+\frac{R \zeta_{x} \phi_{x}}{v^{2}} \\
& -\left(\mu\left(\frac{V_{x}}{v}\right)_{t}-U_{t}^{c}\right) \frac{\phi_{x}}{v}-F_{1} \frac{\phi_{x}}{v}-\frac{P_{x} \phi \phi_{x}}{v^{2}} .
\end{aligned}
$$

Using (36), we obtain by direct calculation

$$
\begin{aligned}
\int_{0}^{t} \int_{\mathbb{R}} F_{1} \frac{\phi_{x}}{v} \mathrm{~d} x \mathrm{~d} t & \leq C \int_{0}^{t}\left\|F_{1}\right\|\left\|\phi_{x}\right\| \mathrm{d} t \leq C_{\eta} \int_{0}^{t}\left\|F_{1}\right\|^{2} \mathrm{~d} t+\eta \int_{0}^{t}\left\|\phi_{x}\right\|^{2} \mathrm{~d} t \\
& \leq C_{\eta} \delta \int_{0}^{t} \mathrm{e}^{-2 c_{0} t} \mathrm{~d} t+\eta \int_{0}^{t}\left\|\phi_{x}\right\|^{2} \mathrm{~d} t \\
& \leq C_{\eta} \delta+\eta \int_{0}^{t}\left\|\phi_{x}\right\|^{2} \mathrm{~d} t .
\end{aligned}
$$

It follows from Lemma 2.4 that

$$
\left|P_{x}\right| \leq C \delta \mathrm{e}^{-c_{0}(|x|+t)},
$$

so, we have

$$
\begin{aligned}
\int_{0}^{t} \int_{\mathbb{R}}\left|\frac{P_{x} \phi \phi_{x}}{v^{2}}\right| \mathrm{d} x \mathrm{~d} t & \leq C\|\phi\|_{L^{\infty}} \int_{0}^{t}\left\|P_{x}\right\|\left\|\phi_{x}\right\| \mathrm{d} t \\
& \leq C_{\eta} \int_{0}^{t}\left\|P_{x}\right\|^{2} \mathrm{~d} t+\eta \int_{0}^{t}\left\|\phi_{x}\right\|^{2} \mathrm{~d} t \\
& \leq C \delta+\eta \int_{0}^{t}\left\|\phi_{x}\right\|^{2} \mathrm{~d} t .
\end{aligned}
$$

The Cauchy inequality leads to

$$
\left|\frac{R \zeta_{x} \phi_{x}}{v^{2}}\right| \leq \eta \phi_{x}^{2}+C_{\eta}\left(\psi_{x}^{2}+\zeta_{x}^{2}\right) .
$$

The estimate (10) and Lemma 2.4 yield that

$$
\begin{aligned}
& \frac{\left|\psi \psi_{x} V_{x}\right|}{v^{2}}+\left|\frac{R \zeta-P \phi}{v^{3}} V_{x} \phi_{x}\right| \\
& \leq \delta \phi_{x}^{2}+C \delta \psi_{x}^{2}+C \delta\left(\phi^{2}+\psi^{2}+\zeta^{2}\right) w^{2}+C \delta \mathrm{e}^{-c_{0}(|x|+t)},
\end{aligned}
$$

and 


$$
\begin{aligned}
& \left|\frac{\psi \phi_{x} U_{x}}{v^{2}}\right|+\left|\left(\mu\left(\frac{V_{x}}{v}\right)_{t}-U_{t}^{c}\right) \frac{\phi_{x}}{v}\right| \\
& \leq C \delta\left((1+t)^{-\frac{1}{2}} w+\left|\psi_{x}\right|+\mathrm{e}^{-c_{0}(|x|+t)}\right)\left|\phi_{x}\right| \\
& \leq C \delta \phi_{x}^{2}+C \delta(1+t)^{-1} w^{2}+C \delta \psi_{x}^{2}+C \delta \mathrm{e}^{-2 c_{0}(|x|+t)} .
\end{aligned}
$$

Lemma 3.3 thus follows directly from Lemma 3.1 and (61), (66)-(72) by first choosing $\eta$ suitably small then $\delta$ suitably small.

Lemma 3.4. Suppose that $(\phi, \psi, \zeta, \omega) \in X([0, T])$ satisfies (31) with suitably small $\varepsilon_{0}+\delta_{0}$. Then it holds for $t \in[0, T]$,

$$
\left\|\psi_{x}(t)\right\|^{2}+\int_{0}^{t}\left\|\psi_{x x}(s)\right\|^{2} \mathrm{~d} s \leq C\left(\delta^{\frac{1}{6}}+\left\|\left(\phi_{0}, \psi_{0}, \zeta_{0}, \omega_{0}\right)\right\|_{1}^{2}\right) .
$$

Proof. Multiplying $(22)_{2}$ by $-\psi_{x x}$, we have

$$
\frac{1}{2}\left(\psi_{x}^{2}\right)_{t}+\mu \frac{\psi_{x x}^{2}}{v}=\left(\psi_{t} \psi_{x}\right)_{x}+\tilde{Q},
$$

with

$$
\begin{aligned}
\tilde{Q}= & \frac{(R \zeta-P \phi)_{x}}{v} \psi_{x x}-\frac{R \zeta-P \phi}{v^{2}} \phi_{x} \psi_{x x}-\frac{R \zeta-P \phi}{v^{2}} V_{x} \psi_{x x} \\
& +\mu \frac{\psi_{x} \phi_{x}}{v^{2}} \psi_{x x}+\mu \frac{\psi_{x} V_{x}}{v^{2}} \psi_{x x}-F \psi_{x x},
\end{aligned}
$$

Now, we need to control the term $\|F\|$. Using (10), (37), Lemma 2.4 and Lemma 2.5, we obtain by direct calculation

$$
\begin{aligned}
& \left|F_{2}^{1}\right| \leq C\left(\left|U_{t}^{c}\right|+\left|U_{x x}^{c}\right|+\left|\left(U_{-}^{r}\right)_{x x}\right|+\left|\left(U_{+}^{r}\right)_{x x}\right|\right)+C \delta \mathrm{e}^{-c_{0}(|x|+t)}, \\
& \left|F_{2}^{2}\right| \leq C \delta \mathrm{e}^{-c_{0}(|x|+t)}, \\
& \left|F_{2}^{3}\right| \leq C\left(\left\|\left(U_{-}^{r}\right)_{x}\right\|_{L^{\infty}}+\left\|\left(U_{+}^{r}\right)_{x}\right\|_{L^{\infty}}+\left\|U_{x}^{c}\right\|_{L^{\infty}}\right)\left|\phi_{x}\right| \leq C \delta^{\frac{1}{2}}(1+t)^{-\frac{3}{4}}\left|\phi_{x}\right| .
\end{aligned}
$$

It follows from (10), (36), (75) and Lemma 2.4 that

$$
\begin{aligned}
\left\|F_{1}\right\| & \leq C \delta \mathrm{e}^{-c_{0} t} \\
\left\|F_{2}^{1}\right\| & \leq C\left(\left\|U_{t}^{c}\right\|+\left\|U_{x x}^{c}\right\|+\left\|\left(U_{-}^{r}\right)_{x x}\right\|+\left\|\left(U_{+}^{r}\right)_{x x}\right\|\right)+C \delta \mathrm{e}^{-c_{0} t} \\
& \leq C \delta(1+t)^{-\frac{5}{4}}+C \delta^{\frac{1}{8}}(1+t)^{-\frac{7}{8}}+C \delta \mathrm{e}^{-c_{0} t}, \\
\left\|F_{2}^{2}\right\| & \leq C \delta \mathrm{e}^{-c_{0} t} \\
\left\|F_{2}^{3}\right\| & \leq C \delta^{\frac{1}{2}}(1+t)^{-\frac{3}{4}}\left\|\phi_{x}\right\| .
\end{aligned}
$$

The estimate (76) yields that

$$
\begin{aligned}
\|F\| & \leq\left\|F_{1}\right\|+\left\|F_{2}^{1}\right\|+\left\|F_{2}^{2}\right\|+\left\|F_{2}^{3}\right\| \\
& \leq C \delta \mathrm{e}^{-c_{0} t}+C \delta(1+t)^{-\frac{5}{4}}+C \delta^{\frac{1}{8}}(1+t)^{-\frac{7}{8}}+C \delta^{\frac{1}{2}}(1+t)^{-\frac{3}{4}}\left\|\phi_{x}\right\| .
\end{aligned}
$$

The Cauchy inequality leads to

$$
\left\|\psi_{x} \phi_{x} \psi_{x x}\right\|_{L^{1}} \leq C\left\|\psi_{x}\right\|^{\frac{1}{2}}\left\|\psi_{x x}\right\|^{\frac{3}{2}}\left\|\phi_{x}\right\| \leq \eta\left\|\psi_{x x}\right\|^{2}+C_{\eta}\left\|\psi_{x}\right\|^{2} .
$$


So, we have

$$
\begin{aligned}
\|\tilde{Q}\|_{L^{1}} \leq & \eta\left\|\psi_{x x}\right\|^{2}+C_{\eta}\left(\left\|\phi_{x}\right\|^{2}+\left\|\psi_{x}\right\|^{2}+\left\|\zeta_{x}\right\|^{2}\right) \\
& +C_{\eta} \delta \int_{\mathbb{R}}\left(\zeta^{2}+\phi^{2}\right) w^{2} \mathrm{~d} x+C_{\eta} \delta^{\frac{1}{4}}(1+t)^{-\frac{7}{4}}+C_{\eta} \delta^{2} \mathrm{e}^{-2 c_{0} t},
\end{aligned}
$$

where we have used

$$
\begin{aligned}
\|F\|^{2} & \leq C \delta^{2} \mathrm{e}^{-2 c_{0} t}+C \delta^{2}(1+t)^{-\frac{5}{2}}+C_{\eta} \delta^{\frac{1}{4}}(1+t)^{-\frac{7}{4}}+C \delta(1+t)^{-\frac{3}{2}}\left\|\phi_{x}\right\|^{2} \\
& \leq C \delta^{2} \mathrm{e}^{-2 c_{0} t}+C \delta^{\frac{1}{4}}(1+t)^{-\frac{7}{4}}+C\left\|\phi_{x}\right\|^{2},
\end{aligned}
$$

due to (77)

Integrating (74) over $\mathbb{R} \times(0, t)$, using (78), and first choosing $\eta$ suitably small then $\delta$ suitably small, we can obtain Equation (73). This completes the proof of Lemma 3.4 .

Lemma 3.5. Suppose that $(\phi, \psi, \zeta, \omega) \in X([0, T])$ satisfies (31) with suitably small $\varepsilon_{0}+\delta_{0}$. Then it holds for $t \in[0, T]$,

$$
\left\|\zeta_{x}(t)\right\|^{2}+\int_{0}^{t}\left\|\zeta_{x x}(s)\right\|^{2} \mathrm{~d} s \leq C\left(\left\|\left(\phi_{0}, \psi_{0}, \zeta_{0}, \omega_{0}\right)\right\|_{1}^{2}+\delta^{\frac{1}{6}}\right) .
$$

Proof. Multiplying Equation (22) $)_{3}$ by $-\zeta_{x x}$, we obtain

$$
\begin{aligned}
& \frac{R}{\gamma-1}\left(\frac{\zeta_{x}^{2}}{2}\right)_{t}+\kappa \frac{\zeta_{x x}^{2}}{v} \\
& =p \psi_{x} \zeta_{x x}+\frac{R \zeta U_{x} \zeta_{x x}}{v}-\frac{P \phi \zeta_{x x} U_{x}}{v}+\kappa \frac{\zeta_{x}\left(\phi_{x}+V_{x}\right) \zeta_{x x}}{v^{2}}+\kappa \frac{\left(\Theta_{x x} \phi+\Theta_{x} \phi_{x}\right) \zeta_{x x}}{v V} \\
& -\kappa \frac{\Theta_{x} \phi\left(\Phi_{x}+V_{x}\right) \zeta_{x x}}{v^{2} V}-\kappa \frac{\Theta_{x} \phi V_{x} \zeta_{x x}}{v V^{2}}-\frac{\omega_{x}^{2} \zeta_{x x}}{v}-v \omega^{2} \zeta_{x x}-G \zeta_{x x} .
\end{aligned}
$$

Now, we are devoted to controlling the term $\left\|G \zeta_{x x}\right\|_{L^{1}}$. Using (10), (44) and Lemma 2.4, we obtain by direct calculation

$$
\left|G_{2}^{1}\right| \leq C \delta \mathrm{e}^{-c_{0}(|x|+t)},
$$

it follows from (43), (46) and (81) that

$$
\left\|G_{1}\right\|+\left\|G_{2}\right\| \leq\left\|G_{1}\right\|+\left\|G_{2}^{1}\right\|+\left\|G_{2}^{2}\right\| \leq C \delta \mathrm{e}^{-c_{0} t} .
$$

Using Lemma 2.2, Lemma 2.4, (31), the Hölder inequality and the Young inequality, we have

$$
\begin{aligned}
\left\|G_{3} \zeta_{x x}\right\|_{L^{1}} \leq & C \int_{\mathbb{R}}\left(U_{x}+\psi_{x}\right)^{2}\left|\zeta_{x x}\right| \mathrm{d} x \leq C \int_{\mathbb{R}}\left(U_{x}^{2}+\psi_{x}^{2}\right)\left|\zeta_{x x}\right| \mathrm{d} x \\
\leq & C \int_{\mathbb{R}}\left(\left(U_{+}^{r}\right)_{x}^{2}+\left(U_{-}^{r}\right)_{x}^{2}+\left(U_{x}^{c}\right)^{2}\right)\left|\zeta_{x x}\right| \mathrm{d} x+C \int_{\mathbb{R}} \psi_{x}^{2}\left|\zeta_{x x}\right| \mathrm{d} x \\
\leq & \eta\left\|\zeta_{x x}\right\|^{2}+C_{\eta} \int_{\mathbb{R}}\left(\left(U_{+}^{r}\right)_{x}^{4}+\left(U_{-}^{r}\right)_{x}^{4}+\left(U_{x}^{c}\right)^{4}\right) \mathrm{d} x+C\left\|\psi_{x}\right\| L_{L^{\infty}}\left\|\psi_{x}\right\|\left\|\zeta_{x x}\right\| \\
\leq & \eta\left\|\zeta_{x x}\right\|^{2}+C_{\eta}\left(\left\|\left(U_{-}^{r}\right)_{x}\right\|_{L^{\infty}}^{2}\left\|\left(U_{-}^{r}\right)_{x}\right\|^{2}+\left\|\left(U_{-}^{r}\right)_{x}\right\|_{L^{\infty}}^{2}\left\|\left(U_{-}^{r}\right)_{x}\right\|^{2}\right. \\
& \left.+\left\|U_{x}^{c}\right\|_{L^{\infty}}^{2}\left\|U_{x}^{c}\right\|^{2}\right)+\eta\left\|\zeta_{x x}\right\|^{2}+C_{\eta}\left\|\psi_{x}\right\|\left\|\psi_{x x}\right\| \\
& \leq \eta\left\|\zeta_{x x}\right\|^{2}+\eta\left\|\psi_{x x}\right\|^{2}+C_{\eta}\left\|\psi_{x}\right\|^{2}+C_{\eta} \delta(1+t)^{-\frac{5}{2}} .
\end{aligned}
$$


The estimate (82) and (83) yields that

$$
\begin{aligned}
\left\|G \zeta_{x x}\right\|_{L^{1}}= & \left\|\left(G_{1}+G_{2}+G_{3}\right) \zeta_{x x}\right\|_{L^{1}} \leq\left\|\left(G_{1}+G_{2}\right) \zeta_{x x}\right\|_{L^{1}}+\left\|G_{3} \zeta_{x x}\right\|_{L^{1}} \\
\leq & \eta\left\|\zeta_{x x}\right\|^{2}+C_{\eta}\left\|G_{1}+G_{2}\right\|^{2}+\left\|G_{3} \zeta_{x x}\right\|_{L^{1}} \\
\leq & \eta\left\|\zeta_{x x}\right\|^{2}+C_{\eta}\left(\left\|G_{1}\right\|+\left\|G_{2}\right\|\right)^{2}+\eta\left\|\zeta_{x x}\right\|^{2}+\eta\left\|\psi_{x x}\right\|^{2} \\
& +C_{\eta}\left\|\psi_{x}\right\|^{2}+C_{\eta} \delta(1+t)^{-3} \\
\leq & \eta\left\|\zeta_{x x}\right\|^{2}+\eta\left\|\psi_{x x}\right\|^{2}+C_{\eta}\left\|\psi_{x}\right\|^{2}+C_{\eta} \delta(1+t)^{-\frac{5}{2}}+C_{\eta} \delta^{2} \mathrm{e}^{-2 c_{0} t} .
\end{aligned}
$$

The Cauchy inequality and Lemma 2.5 lead to

$$
\begin{aligned}
\left\|\frac{\omega_{x}^{2} \zeta_{x x}}{v}\right\|_{L^{1}} & \leq C\left\|\omega_{x}\right\|_{L^{\infty}}\left\|\omega_{x}\right\|\left\|\zeta_{x x}\right\| \leq C\left\|\omega_{x}\right\|^{\frac{1}{2}}\left\|\omega_{x x}\right\|^{\frac{1}{2}}\left\|\zeta_{x x}\right\| \\
& \leq \eta\left\|\zeta_{x x}\right\|^{2}+C_{\eta}\left\|\omega_{x}\right\|\left\|\omega_{x x}\right\| \leq \eta\left\|\zeta_{x x}\right\|^{2}+\eta\left\|\omega_{x x}\right\|^{2}+C_{\eta}\left\|\omega_{x}\right\|^{2},
\end{aligned}
$$

and

$$
\begin{aligned}
\left\|v \omega^{2} \zeta_{x x}\right\|_{L^{1}} & \leq C\|\omega\|_{L^{\infty}}^{2}\left\|\zeta_{x x}\right\|(\|V\|+\|\phi\|) \\
& \leq C\|\omega\|\left\|\omega_{x}\right\|\left\|\zeta_{x x}\right\| \leq \eta\left\|\zeta_{x x}\right\|^{2}+C_{\eta}\left\|\omega_{x}\right\|^{2} .
\end{aligned}
$$

Then, by using the same argument as the previous, and integrating (80) over $\mathbb{R} \times(0, t)$, using (84)-(86) and following Lemma 3.6, and first choosing $\eta$ suitably small then $\delta$ suitably small, one can obtain equation (79). This completes the proof of Lemma 3.5.

The next Lemma is devoted to controlling the term $\int_{0}^{t}\left\|\omega_{x x}(s)\right\|^{2} \mathrm{~d} s$.

Lemma 3.6. Suppose that $(\phi, \psi, \zeta, \omega) \in X([0, T])$ satisfies (31) with suitably small $\varepsilon_{0}+\delta_{0}$. Then it holds for $t \in[0, T]$,

$$
\left\|\omega_{x}(t)\right\|^{2}+\int_{0}^{t}\left\|\omega_{x x}(s)\right\|^{2} \mathrm{~d} s \leq C\left(\left\|\left(\phi_{0}, \psi_{0}, \zeta_{0}, \omega_{0}\right)\right\|_{1}^{2}+\delta^{\frac{1}{6}}\right) .
$$

Proof. Multiplying $(22)_{4}$ by $-\omega_{x x}$, we have

$$
\left(\frac{\omega_{x}^{2}}{2}\right)_{t}+\frac{A}{v} \omega_{x x}^{2}=\frac{A}{v^{2}} \omega_{x} v_{x} \omega_{x x}+A v \omega \omega_{x x} .
$$

We have by Lemma 2.5 (31), the Hölder inequality and the Young inequality that

$$
\begin{aligned}
\left\|\omega_{x} v_{x} \omega_{x x}\right\|_{L^{1}} & =\int_{\mathbb{R}}\left|\omega_{x}\right|\left(\left|V_{x}\right|+\left|\phi_{x}\right|\right)\left|\omega_{x x}\right| \mathrm{d} x \\
& =\int_{\mathbb{R}}\left(\left|\omega_{x} V_{x} \omega_{x x}\right|+\left|\omega_{x} \phi_{x} \omega_{x x}\right|\right) \mathrm{d} x \\
& \leq\left\|V_{x}\right\|_{L^{\infty}} \int_{\mathbb{R}}\left|\omega_{x} \omega_{x x}\right| \mathrm{d} x+\left\|\omega_{x}\right\|_{L^{\infty}}\left\|\phi_{x}\right\|\left\|\omega_{x x}\right\| \\
& \leq \eta\left\|\omega_{x x}\right\|^{2}+C_{\eta}\left\|\omega_{x}\right\|^{2}+C\left\|\omega_{x}\right\|^{\frac{1}{2}}\left\|\omega_{x x}\right\|^{\frac{3}{2}} \\
& \leq \eta\left\|\omega_{x x}\right\|^{2}+C_{\eta}\left\|\omega_{x}\right\|^{2},
\end{aligned}
$$

and

$$
\left\|v \omega \omega_{x x}\right\|_{L^{1}} \leq\left(\|V\|_{L^{\infty}}+\|\phi\|_{L^{\infty}}\right) \int_{\mathbb{R}}\left|\omega \omega_{x x}\right| \mathrm{d} x \leq \eta\left\|\omega_{x x}\right\|^{2}+C_{\eta}\|\omega\|^{2} .
$$


Integrating (88) over $\mathbb{R} \times(0, t)$, using (89) and (90), and first choosing $\eta$ suitably small then $\delta$ suitably small, one can obtain Equation (87). This completes the proof of Lemma 3.6.

\section{Conclusion}

Thus, we finish the proof of Proposition 2.1, and so the proof of Theorem 1.2 is completed.

\section{Conflicts of Interest}

The author declares no conflicts of interest regarding the publication of this paper.

\section{References}

[1] Huang, F.M., Xin, Z.P. and Yang, T. (2008) Contact Discontinuity with General Perturbation for Gas Motions. Advances in Mathematics, 219, 1246-1297. https://doi.org/10.1016/j.aim.2008.06.014

[2] Huang, F.M., Matsumura, A. and Xin, Z.P. (2006) Stability of Contact Discontinuities for the 1-D Compressible Navier-Stokes Equations. Archive for Rational Mechanics and Analysis, 179, 55-77. https://doi.org/10.1007/s00205-005-0380-7

[3] Hsiao, L. and Liu, T. (1993) Nonlinear Diffusive Phenomena of Nonlinear Hyperbolic Systems. Chinese Annals of Mathematics, Series A, 14, 465-480.

[4] Duyn, C.J. and Peletier, L.A. (1977) A Class of Similarity Solutions of the Nonlinear Diffusion Equation. Nonlinear Analysis, 1, 223-233. https://doi.org/10.1016/0362-546X(77)90032-3

[5] Liu, Q.Q. and Yin, H.Y. (2017) Stability of Contact Discontinuity for 1-D Compressible Viscous Micropolar Fluid Model. Nonlinear Analysis, 149, 41-45. https://doi.org/10.1016/j.na.2016.10.009

[6] Smoller, J. (1994) Shock Waves and Reaction-Diffusion Equations. 2nd Edition, Springer-Verlag, New York. https://doi.org/10.1007/978-1-4612-0873-0

[7] Matsumura, A. and Nishihara, K. (1986) Asymptotics toward the Rarefaction Waves of a One-Dimensional Model System for Compressible Viscous Gas. Japan Journal of Applied Mathematics, 3, 1-13. https://doi.org/10.1007/BF03167088

[8] Xin, Z.P. (1996) On Nonlinear Stability of Contact Discontinuities. In: Hyperbolic Problems: Theory, Numerics, Applications, World Scientific, River Edge, 249-257.

[9] Liu, T.P. and Xin, Z.P. (1997) Pointwise Decay to Contact Discontinuities for Systems of Viscous Conservation Laws. The Asian Journal of Mathematics, 1, 34-84. https://doi.org/10.4310/AJM.1997.v1.n1.a3

[10] Xin, Z.P. and Zeng, H.H. (2010) Pointwise Stability of Contact Discontinuity for Viscous Conservation Laws with General Perturbations. Communications in Partial Differential Equations, 35, 1326-1354. https://doi.org/10.1080/03605300903456348

[11] Zeng, H.H. (2009) Stability of a Superposition of Shock Waves with Contact Discontinuities for Systems of Viscous Conservation Laws. Journal of Differential Equations, 246, 2081-2102. https://doi.org/10.1016/j.jde.2008.07.034

[12] Nishihara, K., Yang, T. and Zhao, H.J. (2004) Nonlinear Stability of Strong Rarefaction Waves for Compressible Navier-Stokes Equations. SIAM Journal on Mathematical Analysis, 35, 1561-1597. https://doi.org/10.1137/S003614100342735X 
[13] Matsumura, A. and Nishihara, K. (2000) Global Asymptotics toward the Rarefaction Waves for Solutions of Viscous p-System with Boundary Effect. Quarterly of Applied Mathematics, 58, 69-83. https://doi.org/10.1090/qam/1738558

[14] Matsumura, A. and Nishihara, K. (1992) Global Stability of the Rarefaction Wave of a One-Dimensional Model System for Compressible Viscous Gas. Communications in Mathematical Physics, 144, 325-335. https://doi.org/10.1007/BF02101095

[15] Huang, F.M., Matsumura, A. and Shi, X.D. (2004) On the Stability of Contact Discontinuity for Compressible Navier-Stokes Equations with Free Boundary. Osaka Journal of Mathematics, 41, 193-210.

[16] Huang, F.M., Li, J. and Matsumura, A. (2010) Asymptotic Stability of Combination of Viscous Contact Wave with Rarefaction Waves for One-Dimensional Compressible Navier-Stokes System. Archive for Rational Mechanics and Analysis, 197, 89-116. https://doi.org/10.1007/s00205-009-0267-0

[17] Kawashima, S., Matsumura, A. and Nishihara, K. (1986) Asymptotic Behavior of Solutions for the Equations of a Viscous Heat-Conductive Gas. Proceedings of the Japan Academy, Ser. A, Mathematical Sciences, 62, 249-252. https://doi.org/10.3792/pjaa.62.249

[18] Matsumura, A. and Nishihara, K. (1985) On the Stability of Travelling Wave Solutions of a One-Dimensional Model System for Compressible Viscous Gas. Japan Journal of Industrial and Applied Mathematics, 2, 17-25. https://doi.org/10.1007/BF03167036

[19] Liu, T.P. (1997) Pointwise Convergence to Shock Waves for Viscous Conservation Laws. Communications on Pure and Applied Mathematics, 50, 1113-1182. https://doi.org/10.1002/(SICI)1097-0312(199711)50:11<1113::AID-CPA3>3.0.CO;2$\underline{\mathrm{D}}$

[20] Liu, T.P. and Xin, Z.P. (1988) Nonlinear Stability of Rarefaction Waves for Compressible Navier-Stokes Equations. Communications in Mathematical Physics, 118, 451-465. https://doi.org/10.1007/BF01466726

[21] Kawashima, S. and Matsumura, A. (1985) Asymptotic Stability of Traveling Wave Solutions of Systems for One-Dimensional Gas Motion. Communications in Mathematical Physics, 101, 97-127. https://doi.org/10.1007/BF01212358

[22] Szepessy, A. and Zumbrun, K. (1996) Stability of Rarefaction Waves in Viscous Media. Archive for Rational Mechanics and Analysis, 133, 249-298. https://doi.org/10.1007/BF00380894

[23] Fan, L.L. and Matsumura, A. (2015) Asymptotic Stability of a Composite Wave of Two Viscous Shock Waves for a One-Dimensional System of Non-Viscous and Heat-Conductive Ideal Gas. Journal of Differential Equations, 258, 1129-1157. https://doi.org/10.1016/j.jde.2014.10.010

[24] Fan, L.L., Ruan, L.Z. and Xiang, W. (2018) Asymptotic Stability of a Composite Wave of Two Viscous Shock Waves for the One-Dimensional Radiative Euler Equations. Annales de I Institut Henri Poincaré C, Analyse non linéaire, 36, 1-25. https://doi.org/10.1016/j.anihpc.2018.03.008

[25] Goodman, J. (1986) Nonlinear Asymptotic Stability of Viscous Shock Profiles for Conservation Laws. Archive for Rational Mechanics and Analysis, 95, 325-344. https://doi.org/10.1007/BF00276840

[26] Huang, F.M. and Matsumura, A. (2009) Stability of a Composite Wave of Two Viscous Shock Waves for the Full Compressible Navier-Stokes Equation. Communications in Mathematical Physics, 289, 841-861.

https://doi.org/10.1007/s00220-009-0843-z 
[27] Liu, T.P. (2009) Nonlinear Stability of Shock Waves for Viscous Conservation Laws. Memoirs of the American Mathematical Society, 56, 1-108. https://doi.org/10.1090/memo/0328

[28] Liu, T.P. (1986) Shock Wave for Compressible Navier-Stokes Equations Are Stable. Communications on Pure and Applied Mathematics, 39, 565-594. https://doi.org/10.1002/cpa.3160390502

[29] Liu, T.P. and Zeng, Y.N. (2009) Time-Asymptotic Behavior of Wave Propagation around a Viscous Shock Profile. Communications in Mathematical Physics, 290, 23-82. https://doi.org/10.1007/s00220-009-0820-6

[30] Szepessy, A. and Xin, Z.P. (1993) Nonlinear Stability of Viscous Shock Waves. Archive for Rational Mechanics and Analysis, 122, 53-103. https://doi.org/10.1007/BF01816555

[31] Chen, Z.Z., He, L. and Zhao, H.J. (2015) Nonlinear Stability of Traveling Wave Solutions for the Compressible Fluid Models of Korteweg Type. Journal of Mathematical Analysis and Applications, 422, 1213-1234. https://doi.org/10.1016/j.jmaa.2014.09.050

[32] Chen, Z.Z. and Xiao, Q.H. (2013) Nonlinear Stability of Viscous Contact Wave for the One-Dimensional Compressible Fluid Models of Korteweg Type. Mathematical Methods in the Applied Sciences, 36, 2265-2279. https://doi.org/10.1002/mma.2750

[33] Ma, S.X. and Wang, J. (2016) Decay Rates to Viscous Contact Waves for the Compressible Navier-Stokes Equations. Journal of Mathematical Physics, 57, 1-14. https://doi.org/10.1063/1.4938574

[34] Huang, F.M. and Zhao, H.J. (2003) On the Global Stability of Contact Discontinuity for Compressible Navier-Stokes Equations. Rendiconti del Seminario Matematico della Università di Padova, 109, 283-305.

[35] Hong, H. (2012) Global Stability of Viscous Contact Wave for 1-D Compressible Navier-Stokes Equations. Journal of Differential Equations, 252, 3482-3505. https://doi.org/10.1016/j.jde.2011.11.015

[36] Chen, Z.Z., Xiong, L.J. and Meng, Y.J. (2014) Convergence to the Superposition of Rarefaction Waves and Contact Discontinuity for the 1-D Compressible Navier-Stokes-Korteweg System. Journal of Mathematical Analysis and Applications, 412, 646-663. https://doi.org/10.1016/j.jmaa.2013.10.073

[37] Huang, B.K. and Liao, Y.K. (2017) Global Stability of Viscous Contact Wave with Rarefaction Waves for Compressible Navier-Stokes Equations with Temperature-Dependent Viscosity. Mathematical Models and Methods in Applied Sciences, 27, 2321-2379. https://doi.org/10.1142/S0218202517500464

[38] Huang, F.M. and Wang, T. (2016) Stability of Superposition of Viscous Contact Wave and Rarefaction Waves for Compressible Navier-Stokes System. Indiana University Mathematics Journal, 65, 1833-1875. https://doi.org/10.1512/iumj.2016.65.5914

[39] Duan, R.J., Liu, H.X. and Zhao, H.J. (2009) Nonlinear Stability of Rarefaction Waves for the Compressible Navier-Stokes Equations with Large Initial Perturbation. Transactions of the American Mathematical Society, 361, 453-493. https://doi.org/10.1090/S0002-9947-08-04637-0

[40] Fan, L.L., Liu, H.X., Wang, T. and Zhao, H.J. (2014) Inflow Problem for the One-Dimensional Compressible Navier-Stokes Equations under Large Initial Perturbation. Journal of Differential Equations, 257, 3521-3553. https://doi.org/10.1016/j.jde.2014.07.001

[41] Liu, H.X., Yang, T., Zhao, H.J. and Zou, Q.Y. (2014) One-Dimensional Compressi- 
ble Navier-Stokes Equations with Temperature Dependent Transport Coefficients and Large Data. SIAM Journal on Mathematical Analysis, 46, 2185-2228. https://doi.org/10.1137/130920617

[42] Mujaković, N. (1998) One-Dimensional Flow of a Compressible Viscous Micropolar Fluid: A Local Existence Theorem. Glasnik Matematicki. Serija III, 33, 71-91.

[43] Mujaković, N. (1998) One-Dimensional Flow of a Compressible Viscous Micropolar Fluid: A Global Existence Theorem. Glasnik Matematicki. Serija III, 33, 199-208.

[44] Mujaković, N. (2001) One-Dimensional Flow of a Compressible Viscous Micropolar Fluid: Regularity of the Solution. Radovi Matematički, 10, 181-193.

[45] Mujaković, N. (2007) Non-Homogeneous Boundary Value Problem for One-Dimensional Compressible Viscous Micropolar Fluid Model: A Local Existence Theorem. Annali dell Universita di Ferrara. Sezione VII. Scienze Matematiche, 53, 361-379. https://doi.org/10.1007/s11565-007-0023-z

[46] Mujaković, N. (2008) Non-Homogeneous Boundary Value Problem for One-Dimensional Compressible Viscous Micropolar Fluid Model: Regularity of the Solution. Boundary Value Problems, 15, Article ID: 189748.

https://doi.org/10.1155/2008/189748

[47] Mujaković, N. (2009) Non-Homogeneous Boundary Value Problem for One-Dimensional Compressible Viscous Micropolar Fluid Model: A Global Existence Theorem. Mathematical Inequalities \& Applications, 12, 651-662.

https://doi.org/10.7153/mia-12-49

[48] Mujaković, N. (2010) One-Dimensional Compressible Viscous Micropolar Fluid Model: Stabilization of the Solution for the Cauchy Problem. Boundary Value Problems, 21, Article ID: 796065. https://doi.org/10.1155/2010/796065

[49] Jin, J. and Duan, R. (2017) Stability of Rarefaction Waves for 1-D Compressible Viscous Micropolar Fluid Model. Journal of Mathematical Analysis and Applications, 450, 1123-1143. https://doi.org/10.1016/j.jmaa.2016.12.085

[50] Zheng, L.Y., Chen, Z.Z. and Zhang, S.N. (2018) Asymptotic Stability of a Composite Wave for the One-Dimensional Compressible Micropolar Fluid Model without Viscosity. Journal of Mathematical Analysis and Applications, 468, 865-892. https://doi.org/10.1016/j.jmaa.2018.08.040

[51] Dražić, I. and Mujaković, N. (2012) 3-D Flow of a Compressible Viscous Micropolar Fluid with Spherical Symmetry: A Local Existence Theorem. Boundary Value Problems, 2012, 69. https://doi.org/10.1186/1687-2770-2012-69

[52] Dražić, I. and Mujaković, N. (2014) 3-D Flow of a Compressible Viscous Micropolar Fluid with Spherical Symmetry: Uniqueness of a Generalized Solution. Boundary Value Problems, 2014, 226. https://doi.org/10.1186/s13661-014-0226-Z

[53] Dražić, I. and Mujaković, N. (2015) 3-D Flow of a Compressible Viscous Micropolar Fluid with Spherical Symmetry: Large Time Behavior of the Solution. Journal of Mathematical Analysis and Applications, 431, 545-568. https://doi.org/10.1016/j.jmaa.2015.06.002

[54] Dražić, I. and Mujaković, N. (2015) 3-D Flow of a Compressible Viscous Micropolar Fluid with Spherical Symmetry: A Global Existence Theorem. Boundary Value Problems, 2015, 98. https://doi.org/10.1186/s13661-015-0357-x

[55] Dražić, I., Simčić, L. and Mujaković, N. (2016) 3-D Flow of a Compressible Viscous Micropolar Fluid with Spherical Symmetry: Regularity of the Solution. Journal of Mathematical Analysis and Applications, 438, 162-183.

https://doi.org/10.1016/j.jmaa.2016.01.071 